\title{
Calcineurin in Reactive Astrocytes Plays a Key Role in the Interplay between Proinflammatory and Anti-Inflammatory Signals
}

\author{
Ana M. Fernandez, Silvia Fernandez, Paloma Carrero, Miguel Garcia-Garcia, and Ignacio Torres-Aleman \\ Laboratory of Neuroendocrinology, Cajal Institute, Consejo Superior de Investigaciones Científicas, 28002 Madrid, Spain
}

\begin{abstract}
Maladaptive inflammation is a major suspect in progressive neurodegeneration, but the underlying mechanisms are difficult to envisage in part because reactive glial cells at lesion sites secrete both proinflammatory and anti-inflammatory mediators. We now report that astrocytes modulate neuronal resilience to inflammatory insults through the phosphatase calcineurin. In quiescent astrocytes, inflammatory mediators such as tumor necrosis factor- $\alpha$ (TNF- $\alpha$ ) recruits calcineurin to stimulate a canonical inflammatory pathway involving the transcription factors nuclear factor $\kappa \mathrm{B}(\mathrm{NF} \kappa \mathrm{B})$ and nuclear factor of activated T-cells (NFAT). However, in reactive astrocytes, local anti-inflammatory mediators such as insulin-like growth factor I also recruit calcineurin but, in this case, to inhibit NF $\kappa \mathrm{B} / \mathrm{NFAT}$. Proof of concept experiments in vitro showed that expression of constitutively active calcineurin in astrocytes abrogated the inflammatory response after TNF- $\alpha$ or endotoxins and markedly enhanced neuronal survival. Furthermore, regulated expression of constitutively active calcineurin in astrocytes markedly reduced inflammatory injury in transgenic mice, in a calcineurin-dependent manner. These results suggest that calcineurin forms part of a molecular pathway whereby reactive astrocytes determine the outcome of the neuroinflammatory process by directing it toward either its resolution or its progression.
\end{abstract}

Key words: calcineurin; neuroinflammation; astrocytes; inflammatory cytokines; insulin-like growth factor I; neuronal death

\section{Introduction}

Inflammation, a common denominator among the diverse list of neurodegenerative diseases, has been implicated recently as a key mechanism responsible for the progressive nature of neurodegeneration (Minghetti, 2005). As a result of a low turnover rate and little ability to recover from injury, neurons are extremely vulnerable to self-destructive inflammatory processes. This might hinder the recovery of neurological function at sites of inflammation or even exacerbate neuronal injury through a pathological cascade. The hallmark of brain inflammation is the activation of glial cells (Ridet et al., 1997). These cells constitute essential partners of neurons, providing functional support through a myriad of intercellular links (Seifert et al., 2006). However, during inflammation, glial cells may play a double-edged role because, depending on the course of the inflammatory process, glial-derived factors may result in being either beneficial or detrimental to neurons (Wyss-Coray and Mucke, 2002). Therefore, a balance between proinflammatory and anti-inflammatory signaling arising from a diversity of cells within the affected brain area will eventually determine the outcome of the inflammatory

Received Nov. 8, 2006; revised May 29, 2007; accepted June 14, 2007.

This work was supported by Ministerio de Educacion y Ciencia Grants SAF2001-1722 and 2004-0446 and by Neuropharma. We thank Dr. A. Villarroel (Consejo Superior de Investigaciones Científicas, Bilbao, Spain) for his kind gift of the $\Delta$ CnA plasmid. We appreciate the invaluable help of C. Hernandez with the confocal analysis and C. Lopez-Lopez for technical advice.

Correspondence should be addressed to I. Torres-Aleman, Cajal Institute, Consejo Superior de Investigaciones Científicas, Avenida Dr. Arce 37, 28002 Madrid, Spain. E-mail: torres@cajal.csic.es.

DOI:10.1523/JNEUROSCI.1002-07.2007

Copyright $\odot 2007$ Society for Neuroscience $\quad$ 0270-6474/07/278745-12\$15.00/0 process. If the proinflammatory cascade, mounted mostly by activated microglia and astrocytes, is harnessed, the neuroprotective signals that these two types of glial cells produce, together with those produced by endothelial cells and neurons, will tilt the balance toward resolution of the process. Although the proinflammatory function of astroglia is usually considered not to be as prominent as that of microglia (Streit et al., 1999), astroglia become activated in response to immunologic challenges or brain injuries (Seifert et al., 2006). In fact, reactive astrogliosis is a marker of brain damage in general (Ridet et al., 1997). At the same time, activated astrocytes provide support for damaged neural tissues through several mechanisms, including release of neurotrophic factors and downregulation of proinflammatory messengers (Wyss-Coray and Mucke, 2002). However, the cellular and molecular pathways underlying interactions between proinflammatory and anti-inflammatory signaling within brain tissue are not well delineated.

While analyzing the anti-inflammatory actions of insulin-like growth factor I (IGF-I), a potent neuroprotective signal upregulated in all types of brain lesions (Torres-Aleman, 2000), we observed that inhibition of IGF-I expression at inflammatory sites exacerbated astroglial reactivity (Fernandez et al., 1997). This effect was probably related to the antagonistic actions of IGF-I on tumor necrosis factor- $\alpha$ (TNF- $\alpha$ ) signaling onto astrocytes, an effect in which the phosphatase calcineurin appears to be involved (Pons and Torres-Aleman, 2000). In turn, TNF- $\alpha$ participates in glial-mediated inflammation through activation of the canonical nuclear factor $\kappa \mathrm{B}(\mathrm{NF} \kappa \mathrm{B})$ pathway (Ginis et al., 2002). 
In the present work, we explored proinflammatory and antiinflammatory pathways in astrocytes and their significance in neuronal demise and found that calcineurin plays a dual role in inflammatory responses.

\section{Materials and Methods}

Materials. Human IGF-I was from GroPep (Adelaide, Australia), bacterial lypopolysaccharide (LPS) and rat TNF- $\alpha$ were both from Sigma (St. Louis, MO), the proteasome inhibitor MG-132 (carbobenzoxy-L-leucylL-leucyl-L-leucinal), an IGF-I receptor antagonist [picropodophyllin (PPP)], and the calcineurin inhibitor cyclosporine A (CsA) were purchased from Calbiochem (Darmstadt, Germany). Antibodies against activated caspase 3 (a marker of apoptotic cells; 1:1000; Cell Signaling Technology, Danvers, MA), $\beta 3$-tubulin (a neuronal marker; 1:5000; Promega, Madison, WI), GFAP (an astrocyte marker; 1:1000; Sigma), OX-42 (a microglia marker; 1:400; Serotec, Oxford, UK), A2B5 (an oligodendroglia marker; 1:500; Serotec), major histocompatibility complex II (MHCII) (1:300; Serotec), CD11b (1:500; Serotec), calcineurin (1:1000: Chemicon, Temecula, CA), $\beta$-actin (1:2000; Sigma), superoxide dismutase (SOD) $\mathrm{Cu} / \mathrm{Zn}$ (1:2000; Stressgen, San Diego, CA), inducible nitric oxide synthase 2 (iNOS2) (BD Transduction Laboratories, San Diego, CA), cyclooxygenase 2 (Cox2) (Cayman Chemical, Ann Arbor, MI), IGF-I (GroPep), NF $\kappa$ Bp65, nuclear factor of activated T-cells (NFAT4), GATA3, peroxisome proliferator-activated receptor $\gamma(\operatorname{PPAR} \gamma)$, and ubiquitin (all from Santa Cruz Biotechnology, Santa Cruz, CA), and glyceraldehyde-3-phosphate dehydrogenase (GAPDH) (Affinity BioReagents, Golden, CO) were all used at a 1:1000 dilution except when indicated otherwise. Secondary antibodies were goat anti-rabbit or mouse HRP-coupled (1:20,000; Bio-Rad, Hercules, CA) or Alexacoupled (1:1000; Invitrogen, Carlsbad, CA).

Generation of transgenic mice. Regulated expression of a truncated, constitutively active form of calcineurin $(\Delta \mathrm{CnA})$ in astrocytes was achieved with the "Tet-off" system by generating two transgenic mouse lines. A first mouse line expressed tet-transactivator (tTA) under the astrocyte-specific GFAP promoter (pGFAP). A $1.8 \mathrm{~kb}$ of the murine pGFAP was cloned from genomic tail DNA using primers $5^{\prime}$ CTGTGAACTCGAGACCTGCTT $3^{\prime}$ and $5^{\prime}$ TCTCCGCTGAATTCTGCCCTGC 3' that also generated the sites 5' XhoI and 3' EcoRI, respectively. The pGFAP was placed upstream of a XhoI-EcoRI fragment coding for tTA (pTet-off vector; Clontech, Mountain View, CA). The pGFAP-tTA fragment was cut from the vector by digestion with XhoI and HindIII. A second transgenic line expressed $\Delta \mathrm{CnA}$ under the control of the tTAsensitive TetO promoter. A cDNA encoding a truncated form of the murine calcineurin catalytic subunit $\mathrm{A} \alpha, \Delta \mathrm{CnA}$ (O'Keefe et al., 1992), was placed downstream from the TetO promoter (from pTRE vector; Clontech). The TetO promoter- $\Delta \mathrm{CnA}$ fragment was cut from the vector with XhoI and HindIII. The pGFAP-tTA and TetO- $\Delta$ CnA DNA fragments were purified and independently injected into fertilized eggs of C57BL/6/SJL mice. Founder mice were analyzed by Southern blotting and PCR as described previously (Fernandez et al., 2001), using specific primers and backcrossed to C57BL/6 mice to generate the lines pGFAPtTA (GFAP) and TetO- $\Delta \mathrm{CnA}(\Delta \mathrm{CnA})$. To generate the double-mutant mice [herein called astrocyte inducible calcineurin (AIC) mice], line GFAP7 (F3 generation) was crossed with line $\Delta \mathrm{CnA5}$ (F3 generation). Mice had access to food and plain water ad libitum or water complemented with doxycycline (Dox) $(2 \mathrm{mg} / \mathrm{ml}$; Sigma). Animals were kept under light/dark conditions following European Union guidelines (Directive $86 / 609 / \mathrm{EEC}$ ) and handled according to institutionally approved procedures.

In vivo experiments. AIC mice underwent brain injury following procedures described previously (Lopez-Lopez et al., 2004). Briefly, cortical stab wounds were performed in deeply anesthetized (2,2,2 tribromoethanol; Sigma) mice receiving ( + Dox) or not doxycycline ( - Dox) in their drinking water during 1 week before insult to control astrocyte expression of $\Delta \mathrm{CnA}$. A 26 gauge needle was inserted $3 \mathrm{~mm}$ from the surface of the brain (1.3-1.4 mm caudal to bregma, $1.5 \mathrm{~mm}$ lateral). Animals were allowed to survive for $5,10,20,30$, and $60 \mathrm{~d}$. In a second group of experiments, AIC mice were treated with Dox for 1 week and submitted to brain injury. Two days later, Dox treatment was discontinued in a subset of animals, and the effect of delayed $\Delta \mathrm{CnA}$ expression was determined $5 \mathrm{~d}$ after injury. Another group of animals treated for 1 week with \pm Dox received an LPS injection (Escherichia coli serotype 055:B5; 0.5 $\mu \mathrm{g} / 2 \mu \mathrm{l}$ sterile pyrogen-free saline; Sigma) in the parietal cortex over 2 min with a Hamilton syringe at the following coordinates: $1.3 \mathrm{~mm}$ anteroposterior, $1.5 \mathrm{~mm}$ lateral, and $1 \mathrm{~mm}$ dorsoventral. Animals were allowed to survive for 1,3 , and $10 \mathrm{~d}$. A second group of AIC mice also treated for 1 week before inflammatory challenge with or without Dox received a single intraperitoneal injection of LPS $(1 \mathrm{mg} / \mathrm{kg}$, in $100 \mu \mathrm{l}$ vehicle) or vehicle solution. Animals were allowed to survive for 1 and $5 \mathrm{~d}$. To determine perilesion changes, inflammatory markers and neuronal death were determined by Western blot (WB) and immunofluorescence. For biochemical studies, a block of brain parenchyma surrounding the stab wound or injection site ( $2 \mathrm{~mm}$ wide, $4 \mathrm{~mm}$ high) was dissected from each animal. Ten animals per group were used.

In vitro experiments. Pure astroglial cultures [ $>95 \%$ GFAP-positive $\left(\mathrm{GFAP}^{+}\right), \mathrm{OX}-42$-negative $\left(\mathrm{OX}-42^{-}\right), \mathrm{A}^{\mathrm{B} 5}{ }^{-}$cells] were prepared as described previously (Pons and Torres-Aleman, 2000). Briefly, postnatal (day 3-4) brains were dissected and immersed in ice-cold DMEM/F-12; Invitrogen, Carlsbad, CA) supplemented with $10 \%$ fetal bovine serum (FBS). Cortex and hippocampus were removed and cut into $1 \mathrm{~mm}$ pieces. Tissue fragments were dissociated mechanically, and the resulting cell suspension was centrifuged (1000 rpm/10 min) and plated in DMEM/ F-12 with $10 \%$ FBS, $5 \times 10^{5}$ cells per well. Cerebellar granule cells from 7-d-old rats were grown in plates covered with poly-L-lysine and Neurobasal plus B27 (Invitrogen), glutamine, and $25 \mathrm{~mm} \mathrm{KCl}$. Under these conditions, $\sim 95 \%$ of the cells are neurons $\left(\beta 3\right.$-tubulin $\left.{ }^{+}\right)$. Neurons were plated at $1 \times 10^{6}$ cells per well. Astrocyte-neuron cocultures were prepared as follows: astrocytes were plated, and, when they were $70 \%$ confluent, the medium was removed and neurons were plated onto them with freshly added medium. Cultures were allowed to grow for $4 \mathrm{~d}$. In time course experiments with transfected neuronal cultures or neuronastrocyte cocultures, cells were treated with cytotoxic doses of LPS (E. coli serotype 0127:B8; $1 \mu \mathrm{g} / \mathrm{ml}$; Sigma) or TNF- $\alpha$ (20 ng/ml) for up to 72 and $24 \mathrm{~h}$, respectively. The schedule of the experiments in transfected cocultures is detailed in Figure $1 B$. The calcineurin inhibitor CsA ( $500 \mathrm{~nm})$ was added to transfected astrocytes $24 \mathrm{~h}$ before plating the neurons to avoid inhibition of neuronal calcineurin (see Fig. $1 B$ ). The proteasome inhibitor MG-132 ( $1 \mu \mathrm{M})$ was added $1 \mathrm{~h}$ before LPS or TNF- $\alpha$ stimulation of cocultures (see Fig. 1B). Cultured cells were transfected using Fugene reagent (Roche, Basel, Switzerland) when $70 \%$ confluent. Plasmids transfected were as follows: pGFAP-tTA (GFAP) and TetO- $\Delta \mathrm{CnA}$ $(\Delta \mathrm{CnA})$; pNF $\kappa \mathrm{B}-$ secreted alkaline phosphatase (SEAP) or pNFATSEAP and pCMV- $\Delta \mathrm{CnA}$. Mock transfection was done with pCMV vector.

In experiments using wild-type (wt) cultures, cells were exposed for $16 \mathrm{~h}$ to LPS or for $3 \mathrm{~h}$ to TNF- $\alpha$ before adding IGF-I (100 nM), and cell death was assessed $2 \mathrm{~h}$ later. When investigating the role of calcineurin in IGF-I neuroprotection in wt cells, CsA was added $1 \mathrm{~h}$ before IGF-I. In experiments using cells obtained from AIC mice, doxycycline $(10 \mu \mathrm{g} / \mathrm{ml})$ was added to the cultures at the same time that astrocytes were plated to inhibit $\Delta C n A$ expression from the onset of the experiment.

In vitro assays. Production of reactive oxygen species (ROS) was determined by analyzing $\mathrm{H}_{2} \mathrm{O}_{2}$ levels in the cultures as described previously (Brera et al., 2000), with minor modifications. Briefly, the method uses the nonfluorescent cell-permeant compound $2^{\prime}, 7^{\prime}$-dichlorofluorescein diacetate (Invitrogen) which can be oxidized by peroxides producing the fluorescent compound 2',7'-dichlorofluorescein. Generation of peroxides was measured in a FLUOstar plate reader (BMG Lab technologies, Offenburg, Germany) at an excitation wavelength of $485 \mathrm{~nm}$ and an emission of $520 \mathrm{~nm}$.

To measure the activity of the transcription factors NF $\kappa$ B and NFAT, we used the SEAP reporter system that determines the levels of enzymatically active SEAP. Assays were performed according to the protocol of the manufacturer (Clontech). Transient transfections were performed with $250 \mathrm{ng}$ of the plasmids pNF $\kappa$ B-SEAP and pNFAT-SEAP, and, after $24 \mathrm{~h}$, luminescence was quantified using a Sirius Luminometer (Berthold Detection Systems, Pforzheim, Germany). 
A
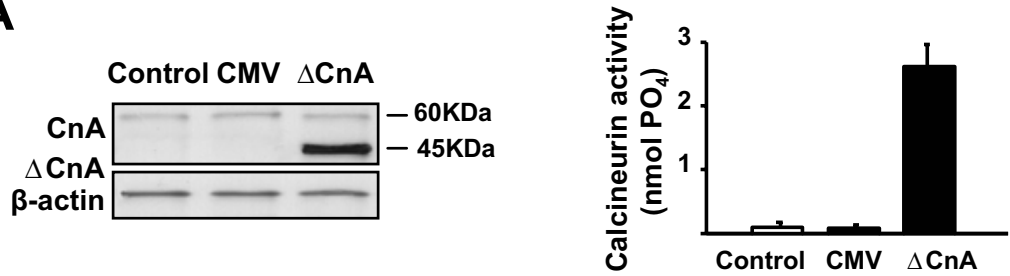

B

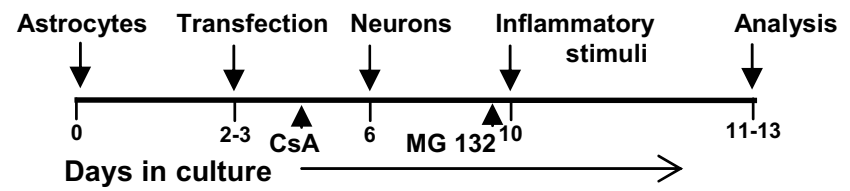

C
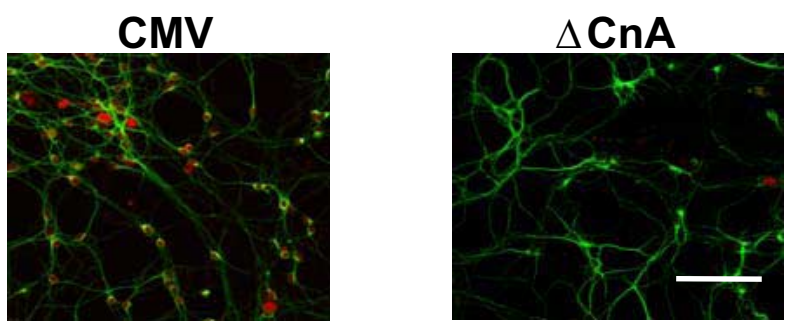

ß3-Tubulin/activated Caspase 3
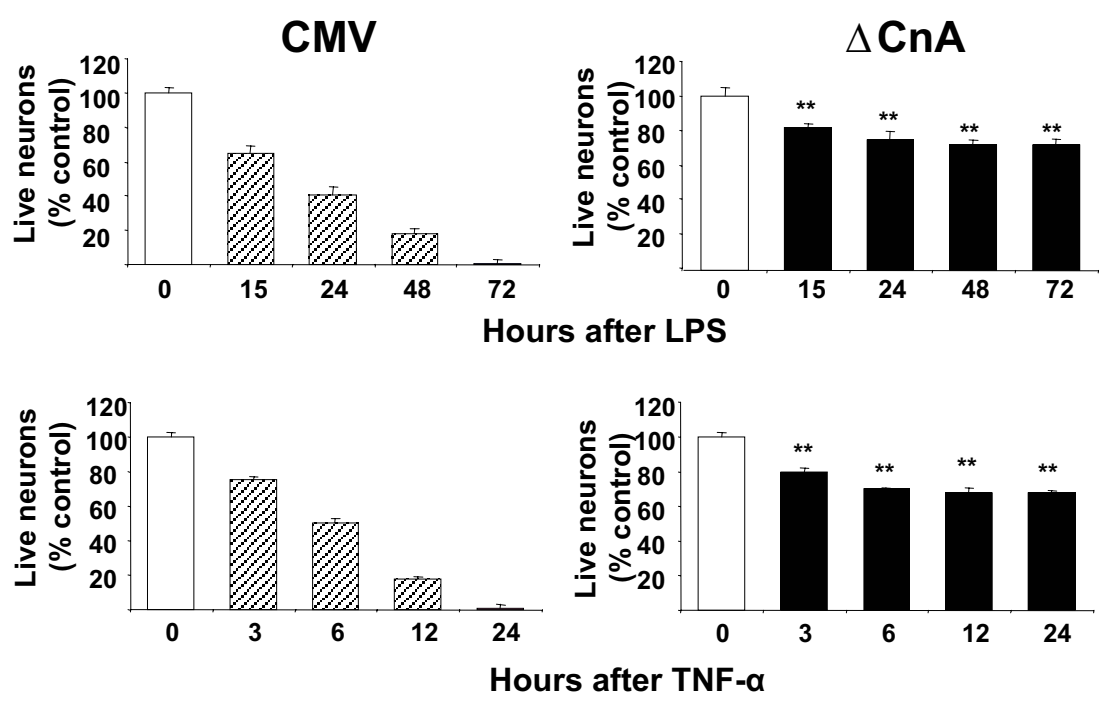

D
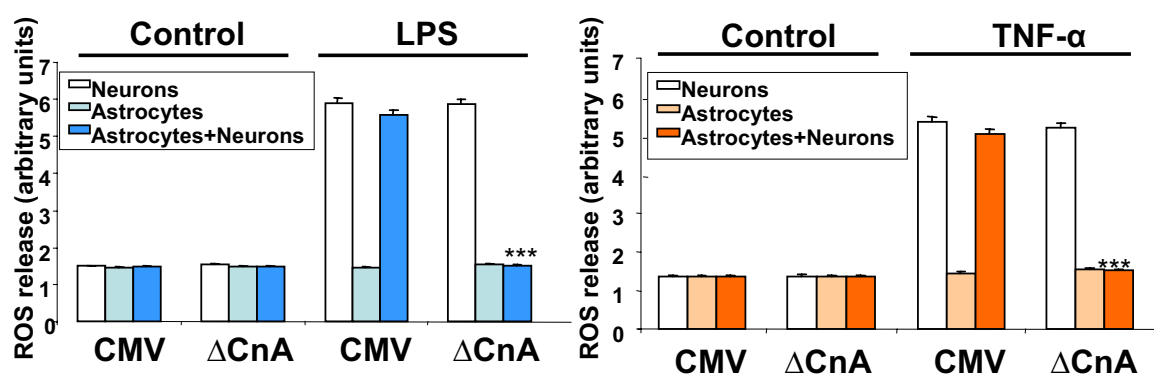

Figure 1. Astrocytic calcineurin and neuroinflammatory damage. $A$, Astrocytes transduced with $\Delta C$ nA show increased activity of calcineurin (measured as release of $\mathrm{PO}_{4}$ ) compared with mock-transfected (CMV) or wt astrocytes (histograms). Only $\Delta \mathrm{CnA}$ transfected astrocytes produced the truncated mutant form of calcineurin $(\triangle \mathrm{CnA}$; blots). Levels of endogenous calcineurin remained unaffected. $\boldsymbol{B}$, Schedule followed for coculture of astrocytes and neurons. Astrocytes were plated and transfected with corresponding DNAs, and neurons were added $24 \mathrm{~h}$ later. Thereafter, cocultures were challenged with inflammatory stimuli (LPS
Calcineurin activity was determined using a calcineurin activity kit (Calbiochem). Culture cells and mouse brain tissue were homogenized in lysis buffer $(50 \mathrm{~mm}$ Tris, $1 \mathrm{~mm}$ DTT, $100 \mu \mathrm{M}$ EDTA, $100 \mu \mathrm{M}$ EGTA, and 0.2\% NP-40, pH 7.5) and protease inhibitor cocktail (Roche). To remove free phosphate, supernatant extracts were added to a desalting column resin. Phosphatase assays were performed in a total of 100 $\mu \mathrm{l}$ in assay buffer $(200 \mathrm{~mm} \mathrm{NaCl}, 100 \mathrm{~mm}$ Tris, $12 \mathrm{~mm} \mathrm{MgCl}_{2}, 1 \mathrm{~mm} \mathrm{CaCl}, 1 \mathrm{~mm}$ DTT, and $0.05 \%$ NP-40, pH 7.5). Recombinant human calcineurin at $50 \mathrm{U} / \mu \mathrm{l}, 25 \mu \mathrm{M}$ calmodulin, and $10 \mu \mathrm{l}$ RII phosphopeptide were used per assay. When indicated, EGTA was added $(20 \mathrm{~mm})$, and assays were incubated at $30^{\circ} \mathrm{C}$ for $30 \mathrm{~min}$. Calcineurin phosphatase activity was measured by luminescent detection at $620 \mathrm{~nm}$ of free phosphate released from the calcineurinspecific RII phosphopeptide using the green reagent. Assays were performed in duplicate at each time point in three separate experiments.

Reverse transcription-PCR was performed using the QuantumRNA Universal 18 S system from Ambion (Austin, TX). Total RNA was isolated from cultured cells or mouse tissues using Trizol (Invitrogen). Equivalent amounts of total RNA served as template for cDNA synthesis using M-MLV (Moloney murine leukemia virus) reverse transcriptase (Promega) at $42^{\circ} \mathrm{C}$, followed by PCR using specific primers directed to the $3^{\prime}$ end of the GFAP promoter and the $5^{\prime}$ end of $\triangle \mathrm{CnA}$ : forward, 5' GATGTGCTTTACTAAGTCATC 3'; and reverse, 5' CAGTTCATCGTCGGAGCAGA $3^{\prime}$. RNA 18 S was quantitated as a loading control.

For RNA interference experiments [Silencer small interference RNA (siRNA) transfection II kit; Ambion], three different double-stranded CnA siRNAs (with sequences CnA1, 5' GGAAGUGUUUGAUAACGAU 3'; CnA2, 5' GGAAGGCAGGCUGGAAGAA 3'; CnA3, 5' GGAUUCUCACCACAACAU $3^{\prime}$ ) were transfected (30 $\mathrm{nM}$ ) with siPORT NeoFX (Ambion) into primary astrocytes (12-well plates containing $9 \times 10^{4}$ cells $/ \mathrm{ml}$ ). Seventy-two hours later, cultures were

\section{$\leftarrow$}

or TNF- $\alpha$ ) for various times before analysis of neuronal death after a total of $4 \mathrm{~d}$ in coculture. Inhibitors such as CsA or MG132 were added at indicated times. C, Photomicrographs, Representative double immunocytochemical staining used to identify apoptotic (activated caspase $3^{+}$cells; red) neurons $\left(\beta 3\right.$-tubulin ${ }^{+}$cells; green) after inflammatory challenge. Histograms, Neurons cocultured with mock-transfected astrocytes (CMV; striped left histograms) die soon after LPS (top histograms) or TNF- $\alpha$ addition (bottom histograms), whereas when cocultured with astrocytes expressing $\Delta C n A$ (black right histograms), they show a significantly greater resistance to these inflammatory stimuli $\left({ }^{* *} p<0.01\right.$ vs respective (MV times). Number of living neurons are expressed as percentage of unstimulated control cultures at time 0 . Scale bar, $100 \mu \mathrm{m}$. D, The release of ROS in response to proinflammatory stimuli (LPS or TNF- $\alpha$ ) was fully abrogated when astrocytes (but not neurons) expressed $\Delta$ CnA but not the empty vector (CMV). Note that, with the doses of LPS or TNF- $\alpha$ used, astrocytes did not produce ROS. All experiments were repeated six times. ${ }^{* * *} p<0.001$. 
A

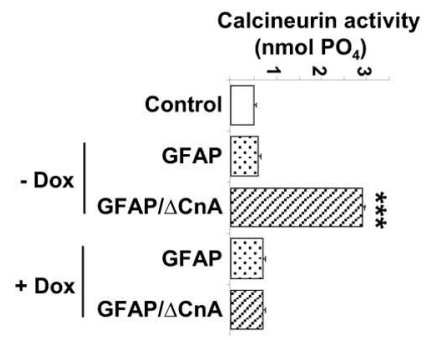

B

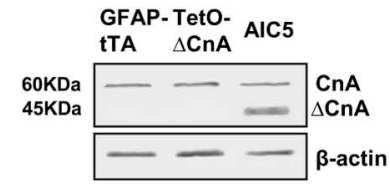

C

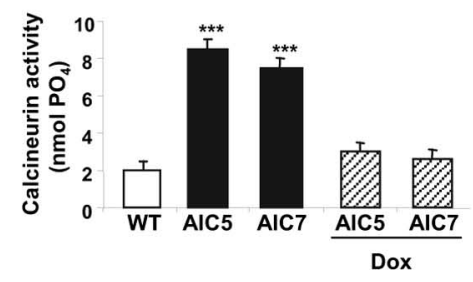

D

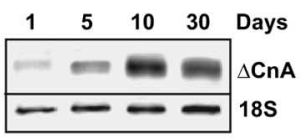

E

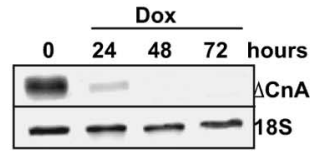

$\mathbf{F}$

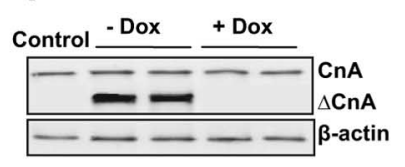

G

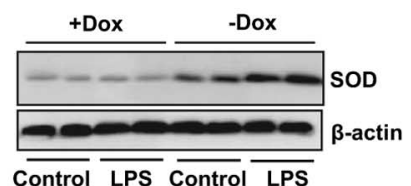

H

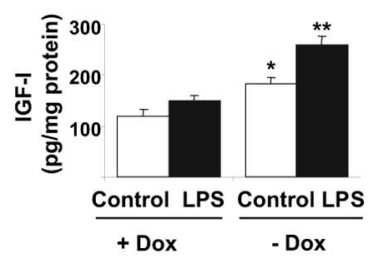

Figure 2. Regulated expression of $\Delta C n A$ in astrocytes. $A$, Coexpression of GFAP-tTA (GFAP) and Tet0- $\Delta C \mathrm{Cn}(\Delta \mathrm{CnA})$ in cultured wt astrocytes allows Dox-regulated activation of calcineurin. Both control (wt) and GFAP-tTA-transduced astrocytes show lower and Doxindependent calcineurin activity. ${ }^{* *} p<0.001$. B, Astrocyte inducible calcineurin (AIC mouse 5) but not single mutant Tet $0-\Delta C$ nA or GFAP-tTA mice produced $\Delta C$ nA protein, a truncated form of the wt protein. Wild-type calcineurin $(\mathrm{CnA})$ was detected in the three animals. C, Calcineurin activity in brain extracts from AIC mice (lines 5,7 ) was markedly elevated compared with a wt control and was regulated by $D o x .{ }^{* * *} p<0.001$. D, Expression of $\Delta C n A$ mRNA in the brain of AIC mice was found postnatally and maintained thereafter. $\boldsymbol{E}, \Delta C \mathrm{Cn}$ mRNA levels in the brain of AIC mice were inhibited within hours after addition of Dox to the drinking water. $\boldsymbol{F}$, Expression of $\Delta C n A$ protein in astrocytes obtained from AIC mice was regulated by Dox in the culture medium. Controls are astrocytes from wt mice. $\mathbf{G}$, Astrocytes from AIC mice secrete $\mathrm{Cu} / \mathrm{Zn} \mathrm{SOD} \mathrm{(SOD)} \mathrm{to} \mathrm{the} \mathrm{medium} \mathrm{after} \mathrm{LPS} \mathrm{challenge} \mathrm{in} \mathrm{a} \mathrm{calcineurin-dependent} \mathrm{manner.} \mathrm{In} \mathrm{the}$ presence of Dox in the culture medium (which suppresses $\Delta C n A$ expression), levels of SOD remain unaffected. Note that expression of $\Delta C n A$ is sufficient to increase SOD levels. Representative blot is shown $(n=6)$. $\boldsymbol{H}$, AIC astrocytes also secrete greater amounts of IGF-I after LPS in the absence of Dox in the culture medium $\left({ }^{*} p<0.05\right.$ vs control and ${ }^{* *} p<0.01$ vs + Dox and vs control; $n=6$ ). stimulated with LPS ( $1 \mu \mathrm{g} / \mathrm{ml})$ overnight. Cells were lysed, and levels of CnA, iNOS2, Cox2, GAPDH (as a siRNA internal control), and $\beta$-actin were determined by WB. A nontargeting siRNA and a GAPDH siRNA were used as negative and positive controls, respectively. Assays were performed in triplicate.

Immunofluorescence. Immunocytochemistry was performed as described previously (Trejo et al., 2001). Animals ( $n=10$ per group) were deeply anesthetized with pentobarbital $(50 \mathrm{mg} / \mathrm{kg})$ and perfused transcardially with $4 \%$ paraformaldehyde in $0.1 \mathrm{~m}$ phosphate buffer, $\mathrm{pH} 7.4$ (PB). Coronal $40-\mu \mathrm{m}$-thick sections were cut in a vibratome and collected in PBS. For in vitro experiments, cells were plated on $20 \mathrm{~mm}$ coverslips and fixed. Coverslips or free-floating brain sections were blocked with $5 \%$ normal horse serum and incubated overnight at $4{ }^{\circ} \mathrm{C}$ with the respective primary antibody in $\mathrm{PB}$ containing $0.1 \%$ bovine albumin, $3 \%$ horse serum, and $0.2 \%$ Triton X-100. After several washes in $\mathrm{PB}$, sections were incubated with an Alexa-coupled secondary antibody (1:1000). Omission of primary antibody was used as control. Neuronal death was determined by a single investigator in uncoded brain sections and cultures, as described (Fernandez et al., 1998). $\beta 3$-Tubulin ${ }^{+} /$acti- $^{-}$ vated caspase $3^{+}$double-stained cells were scored and expressed as percentage of total $\beta 3$-tubulin ${ }^{+}$cells. Activated caspase $3^{+}$neurons were scored in six consecutive sections in a $1 \mathrm{~mm}^{2}$ area or in six different microscope fields for each coverslip when counting cell cultures. $\mathrm{MH}-$ $\mathrm{CII}^{+}$and $\mathrm{CD} 11 \mathrm{~b}^{+}$cells in double-stained brain sections from lesioned areas were scored and expressed as number of cells per square millimeter. Confocal analysis was performed in a Leica (Bensheim, Germany) microscope.

Immunoassays. Animals were perfused transcardially with saline before collection of brain samples for biochemical analysis. Cells or cerebral cortex pieces ( $1 \mathrm{~mm}$ around lesion or injection) were homogenized in ice-cold buffer consisting of $20 \mathrm{~mm}$ Tris $\mathrm{HCl}, 150 \mathrm{~mm} \mathrm{NaCl}, 5 \mathrm{~mm}$ EDTA, $10 \%$ glycerol, $1 \% \mathrm{NP}-40,400 \mu \mathrm{M}$ sodium orthovanadate, and protease inhibitors (200 mM PMSF, $1 \mu \mathrm{g} / \mathrm{ml}$ aprotinin, and $1 \mu \mathrm{g} / \mathrm{ml}$ leupeptin). $\mathrm{WB}$ and immunoprecipitation were performed as described previously (Pons and Torres-Aleman, 2000). WB membranes were reblotted with either the same antibody used for immunoprecipitation or with $\beta$-actin as internal standards and to normalize for protein load. Levels of the protein under study were expressed relative to protein load in each lane. Densitometric analysis was performed using Quantity One software (Bio-Rad). IGF-I was quantified in cell lysates and media by sandwich ELISA using mouse IGF-I as a standard (IBT, Reutlingen, Germany). Briefly, 96-well plates were covered with a monoclonal anti-mouse IGF-I antibody (1:1000; R \& D Systems, Minneapolis, $\mathrm{MN})$ at $4^{\circ} \mathrm{C}$ overnight. After several washes with PBS, pH 7.4, wells were incubated with a blocking solution containing PBS, pH 7.4, 5\% Tween 20, 5\% sucrose, and $0.05 \%$ sodium azide, $2 \mathrm{~h}$ at room temperature. After three washes with PBS, pH 7.4, plates were placed at $4^{\circ} \mathrm{C}$ overnight. Samples were added and maintained at $4^{\circ} \mathrm{C}$ overnight. After washes, plates were incubated with a biotinylated anti-mouse IGF-I antibody (1:1000; R \& D Systems) at $4^{\circ} \mathrm{C}$ overnight. After washes, plates were incubated with a streptavidin horseradish peroxidase conjugate (1:20,000; GE Healthcare, Little Chalfont, UK). After washing, $o$-phenyllenediamine dihydrochloride (Sigma) was added during $30 \mathrm{~min}$ at room temperature. Absorbance was measured in a spectrophotometer at $450 \mathrm{~nm}$.

A protein array (1.1; Raybiotech, Norcross, GA) to quantitate relative expression of 40 different inflammation-related factors was used following the instructions of the manufacturer. Membranes were blocked for 30 min and incubated with $50 \mu \mathrm{g}$ of AIC astrocyte culture lysate or with 500 $\mu \mathrm{g}$ of AIC tissue homogenate for $2 \mathrm{~h}$ at room temperature and then washed for $30 \mathrm{~min}$ and incubated with a biotin-conjugated antibody (1:250) for $2 \mathrm{~h}$. After consecutive washes, streptavidin-conjugated peroxidase $(1: 1000)$ was added for $1 \mathrm{~h}$ at room temperature. Membranes were washed thoroughly and exposed to ECL substrate (GE Healthcare), followed by apposition of the membranes with autoradiographic film (GE Healthcare) for $1 \mathrm{~min}$. The film was scanned, and spots were digitized into pixel densities using the Quantity One imaging software (BioRad). The ratio of relative expression was established after subtraction of the background intensity and comparison with the positive spots available in the membrane. 
A

\section{B3-Tubulin/activated caspase 3}
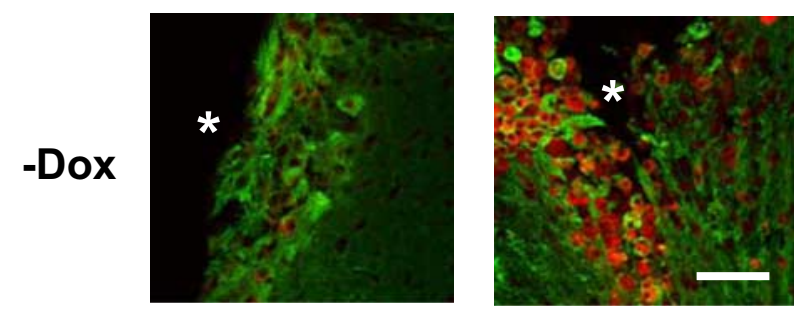

\section{+ Dox}

B
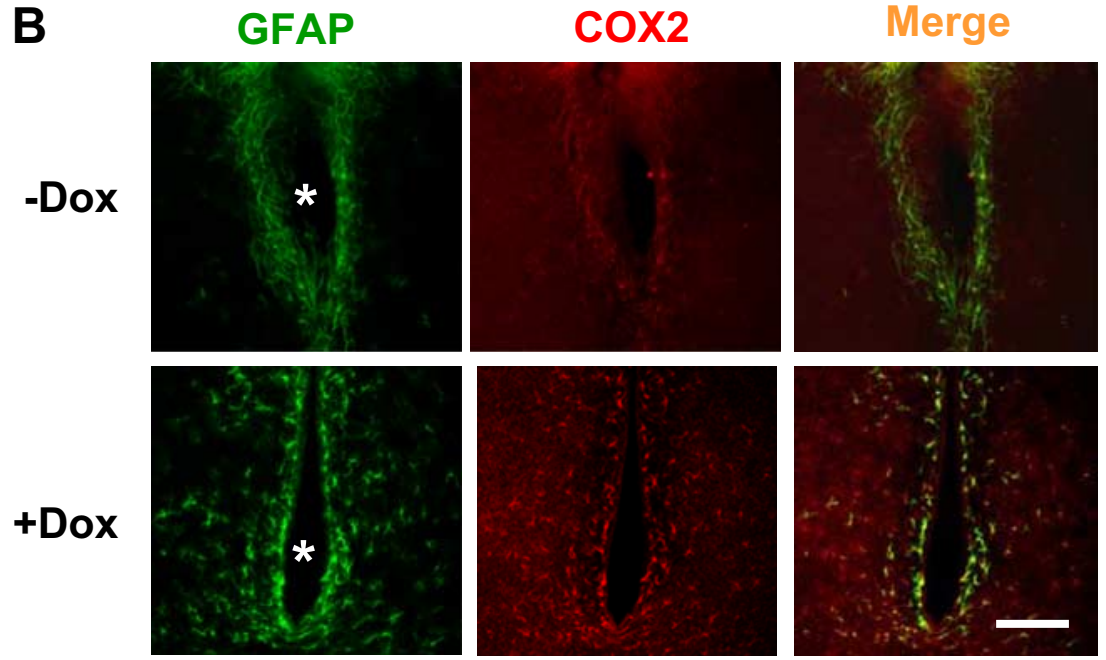

C
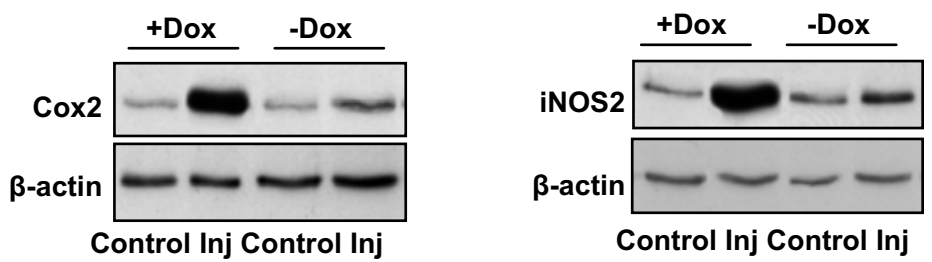

D
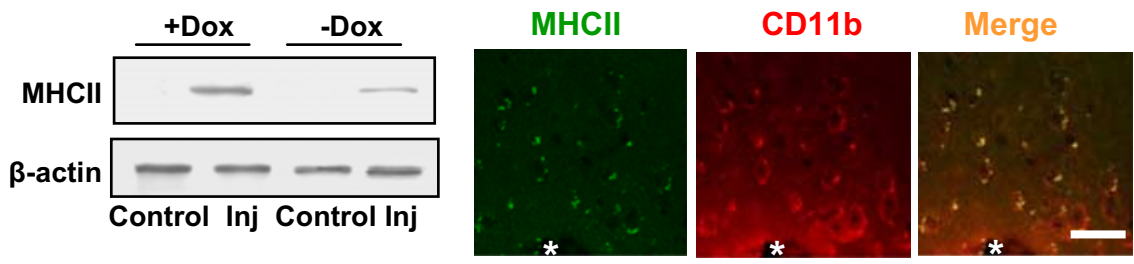

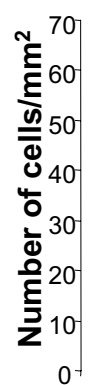
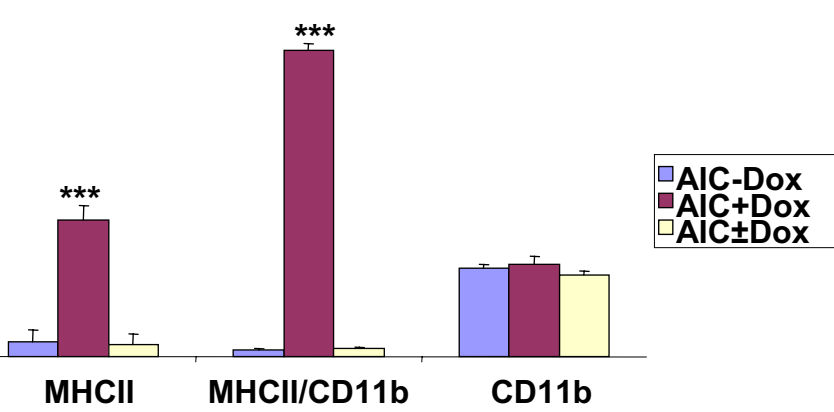

Figure 3. Astrocytic calcineurin protects against inflammatory damage after brain trauma. $A$, In AIC mice expressing $\Delta C n A$ (not treated with Dox), the number of dying neurons ( $\beta 3$-tubulin ${ }^{+}$-activated caspase $3^{+}$cells) $5 \mathrm{~d}$ after the lesion in the area surrounding a traumatic injury (asterisk) of the parietal cortex was reduced compared with AIC mice treated with Dox for 1 week before lesion. Scale bar, $50 \mu \mathrm{m} . \boldsymbol{B}$, Expression of $\Delta \mathrm{CnA}$ in AIC mice ( - Dox) reduced the number of reactive astrocytes (GFAP ${ }^{+}$; green) expressing Cox2 (red) in the lesioned area (asterisk). Note expression of Cox2 also by unidentified GFAP $^{-}$cells
Statistics. Statistical analyses were performed with a $t$ test when comparing two groups and a two-way ANOVA for comparing multiple groups. Statistical significance was established when $p<0.05$. Results shown are mean \pm SEM.

\section{Results}

Calcineurin participates in astrocytemediated neuroprotection against inflammatory damage

As mentioned above, we previously found that the phosphatase calcineurin mediates the anti-inflammatory effects of IGF-I on astrocytes (Pons and Torres-Aleman, 2000). To gain additional insight into the biological significance of this antiinflammatory activity, we transfected wt rat astrocytes with a constitutively active form of the catalytic subunit of calcineurin $(\Delta \mathrm{CnA})$. Transfected astrocytes showed high levels of $\triangle \mathrm{CnA}$ together with marked increases in calcineurin activity (Fig. $1 A$ ). Next, we tested the neuroprotective activity of $\Delta$ CnA-transfected astrocytes against inflammatory damage. When rat cerebellar neurons were cocultured with mocktransfected astrocytes [cytomegalovirus (CMV)-transfected astrocytes] and submitted to TNF- $\alpha$ or LPS challenge, they died within $24-72 \mathrm{~h}$, respectively (Fig. 1C, left histograms). However, when neurons were cocultured with astrocytes expressing $\triangle \mathrm{CnA}$, neuronal death after either LPS (1 $\mu \mathrm{g} / \mu \mathrm{ml})$ or TNF- $\alpha(20 \mathrm{ng} / \mathrm{ml})$ was markedly abrogated (Fig. $1 C$, right histograms) ( $p<0.01$ vs CMV-transfected astrocytes). Neuroprotection was mediated at least in part by humoral factors because conditioned medium from $\Delta \mathrm{CnA}$-expressing

\section{$\leftarrow$}

surrounding the lesion site. Scale bar, $100 \mu \mathrm{m}$. C, Levels of both Cox2 and iNOS2 at the injury site were also dramatically reduced in $\Delta$ CnA-expressing AIC mice ( $p<0.001$ vs AIC + Dox, for both markers; $n=10$ at $5 \mathrm{~d}$ after lesion). Control, Sham operated; Inj, brain injury. D, Levels of MHCII (a marker of microglia activation) were increased at the lesion site $5 d$ after brain trauma in AIC mice not expressing $\Delta \mathrm{CnA}$ ( + Dox; $n=10$ ), but the increase was smaller when expressing $\Delta C n A$ $(-$ Dox; $n=10)$. Protein load in gels was normalized by measuring $\beta$-actin levels. Bottom histograms, Number of $\mathrm{MH}$ $\mathrm{ClI}^{+}, \mathrm{CD}_{11 \mathrm{~b}}{ }^{+}$(a marker of infiltrating macrophages), and double $\mathrm{MHCll}^{+}-\mathrm{CD}_{11 \mathrm{~b}^{+}}$cells at the lesioned site in AIC mice treated with Dox 1 week before lesion (+Dox), not treated with Dox ( - Dox), or treated with Dox until $2 \mathrm{~d}$ after the lesion ( \pm Dox). In the absence of Dox, when $\Delta C n A$ is expressed in astrocytes, the number of $\mathrm{MHCll}^{+}$and $\mathrm{MHCll}^{+}{ }_{-}$ $\mathrm{CD}_{11 \mathrm{~b}}{ }^{+}$cells is significantly reduced, whereas $\mathrm{CD} 11 \mathrm{~b}{ }^{+}$cells remain unaffected. Note that the reduction is also present when AIC mice started to express $\Delta \mathrm{CnA} 2 \mathrm{~d}$ after the lesion was produced ${ }^{* * *} p<0.001$ vs - Dox and \pm Dox; $n=5$ per group). Photomicrographs, Representative $\mathrm{MHClI}^{+}$and $\mathrm{CDb}_{11}{ }^{+}$cells located in the vicinity of the lesion site (asterisk). Cell counts were done in double-stained brain sections. Scale bar, $50 \mu \mathrm{m}$. 


\begin{tabular}{|c|c|c|c|}
\hline Cytokine & Brain trauma & Brain LPS & In vitro LPS \\
\hline BLC & 5 & 4 & 2 \\
\hline Eotaxin & 5 & 4 & 2 \\
\hline GM-CSF & 5 & 4 & 2 \\
\hline IFN $\gamma$ & 5 & 4 & 2 \\
\hline IL-1 $\alpha$ & 4 & 4 & 3 \\
\hline IL-1 $\beta$ & 4 & 4 & 3 \\
\hline IL-2 & 4 & 4 & 3 \\
\hline IL-3 & 4 & 4 & 3 \\
\hline IL-4 & 4 & 4 & 3 \\
\hline IL-8 & 4 & 5 & 3 \\
\hline IL-9 & 4 & 5 & 3 \\
\hline IL-10 & 4 & 5 & 3 \\
\hline IL-12 $2^{\text {40p70 }}$ & 4 & 5 & 3 \\
\hline $\mathrm{IL}-12^{\mathrm{p} 70}$ & 4 & 5 & 3 \\
\hline IL-13 & 5 & 5 & 3 \\
\hline |L-17 & 5 & 5 & 3 \\
\hline RANTES & 5 & 4 & 2 \\
\hline TIMP-1 & 5 & 4 & 2 \\
\hline TIMP-2 & 5 & 4 & 2 \\
\hline TNF- $\alpha$ & 3 & 4 & 3 \\
\hline sTNF RI & 3 & 4 & 3 \\
\hline sTNF RII & 3 & 4 & 3 \\
\hline
\end{tabular}

Numbers indicate fold increase over respective -Dox groups. Results are the mean of two membranes for each treatment and condition. Unchanged cytokines are not shown (supplemental Fig. 2B, available at www. jneurosci.org as supplemental material). BLC, B-lymphocyte chemoattractant; GM-CSF, granulocyte macrophage

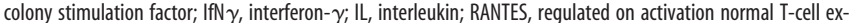
pressed and secreted; TIMP, tissue inhibitor of metalloproteinase; sTNF RI and RII, soluble TNF receptors I and II.

astrocytes protected neurons against inflammatory damage after $16 \mathrm{~h}$ of exposure to LPS $(36 \pm 4 \%$ of neurons alive in conditioned medium from $\Delta \mathrm{CnA}$ astrocytes vs $1 \pm 1.2 \%$ neurons alive in conditioned medium from CMV astrocytes; $p<$ 0.05 ) or $3 \mathrm{~h}$ after TNF- $\alpha$ (data not shown). This protective action was not observed when neurons themselves expressed $\Delta \mathrm{CnA}$ (supplemental Fig. $1 \mathrm{~A}$, available at www.jneurosci.org as supplemental material). At the doses used, LPS or TNF- $\alpha$ did not affect astrocyte survival (data not shown). In addition, when calcineurin activity was inhibited in $\Delta \mathrm{CnA}$-expressing astrocytes by adding the calcineurin inhibitor CsA $24 \mathrm{~h}$ before coculturing them with neurons, neuroprotection against LPS or TNF- $\alpha$ (data not shown) was significantly diminished ( $72 \pm 5 \%$ of neurons alive compared with $35 \pm 5 \%$ alive when CsA was added; $p<0.05)$. Furthermore, neuronal production of ROS in response to LPS or TNF- $\alpha$ challenge was completely abrogated when neurons were cocultured with $\Delta \mathrm{CnA}$ expressing astrocytes but not when neurons were cultured alone or with mock-transfected astrocytes ( $p<0.001$ vs CMV transfected) (Fig. 1D).

\section{Astrocyte calcineurin protects against brain inflammatory injury}

We next designed in vivo"proof-of-concept" experiments to establish an anti-inflammatory action of calcineurin in astrocytes. Double transgenic mice with Dox-regulated expression of $\Delta \mathrm{CnA}$ in astrocytes were generated by crossbreeding mice expressing a murine GFAP promoter-driven tTA transgene with mice expressing a TetO-driven $\Delta \mathrm{CnA}$ transgene. We first corroborated that in vitro expression of these transgenes allowed Doxregulated calcineurin activity in wt astrocytes cotransfected with them (Fig. 2A). In vivo, double transgenic AIC mice expressed $\triangle \mathrm{CnA}$ in the brain (Fig. $2 B$ ) and markedly enhanced calcineurin activity in a Dox-regulated manner (Fig. 2C). Detailed characterization of AIC mice confirmed the expression of the two transgenes (GFAP-tTA and TetO- $\Delta \mathrm{CnA}$ ) (supplemental Fig. $1 B$, available at www.jneurosci.org as supplemental material), expression of $\triangle \mathrm{CnA}$ mRNA in the brain (supplemental Fig. $1 C$, available at www.jneurosci.org as supplemental material), and Dox-regulated calcineurin immunoreactivity in astrocytes (supplemental Fig. $1 D$, available at www.jneurosci.org as supplemental material). Expression of $\Delta \mathrm{CnA}$ mRNA in AIC brain appeared early after birth and was maintained thereafter (Fig. 2D).

Importantly, when AIC mice received Dox in the drinking water, $\triangle \mathrm{CnA}$ mRNA was inhibited within $24-48 \mathrm{~h}$ (Fig. $2 E$ ). Because expression of $\Delta \mathrm{CnA}$ in cultured AIC astrocytes was also inhibited by adding Dox $(10 \mu \mathrm{g} / \mathrm{ml})$ at the onset of the culture (Fig. 2 F), we concluded that calcineurin activity in AIC astrocytes was regulated by Dox in vivo and in vitro. That calcineurin activity is regulated by Dox was indirectly confirmed by the observation that Dox regulated the release by AIC astrocytes of humoral neuroprotective signals such as $\mathrm{Cu} / \mathrm{Zn} \mathrm{SOD}$ or IGF-I that are known to be functionally associated with calcineurin (Tokheim and Martin, 2006; Alfieri et al., 2007). Thus, in response to proinflammatory challenge with LPS, AIC astrocytes secreted larger amounts of SOD and IGF-I when cultured without Dox (Fig. $2 G, H)$. Similar results were obtained in wild-type rat astrocytes transduced with $\Delta \mathrm{CnA}$ and challenged with LPS (supplemental Fig. $1 E$, available at www.jneurosci.org as supplemental material) or TNF- $\alpha$ (data not shown), respectively. When the IGF-I receptor antagonist PPP (120 nM) was added to LPS-stimulated cocultures containing $\Delta \mathrm{CnA}$-expressing astrocytes, the neuroprotective action of astrocytes was moderately reduced: $\sim 70 \%$ of the neurons were alive in $\Delta \mathrm{CnA}$-expressing astrocyte cocultures, whereas after adding PPP, $\sim 60 \%$ of neurons were alive. However, in cocultures with mock-transfected astrocytes, the presence of PPP reduced to $50 \%$ the neuronal survival induced by astrocytes after LPS insult (from $\sim 30$ to $\sim 15 \%$ neurons alive).

Once we observed that calcineurin protects neurons against inflammatory death in vitro, we explored whether calcineurin in AIC astrocytes protected neurons against brain inflammation in vivo. AIC mice submitted to a penetrating brain injury that produces an inflammatory response in the lesioned area were analyzed 5, 10, 20, 30, and 60 d later. At $60 \mathrm{~d}$, weak glial reactivity was still present in AIC mice not expressing $\Delta \mathrm{CnA}$, but the rest of the changes analyzed were resolved within 1 month. Because all pathological changes examined peaked within $5-10 \mathrm{~d}$ of the lesion, we analyzed them in detail $5 \mathrm{~d}$ after the lesion. In AIC mice with suppressed $\Delta \mathrm{CnA}$ expression by treatment with Dox ( + Dox AIC mice), neuronal death (identified as double-stained $\beta 3$ tubulin ${ }^{+}$-activated caspase $3^{+}$cells) $5 \mathrm{~d}$ after brain injury was prominent (Fig. $3 A$ ). Dox-treated AIC mice also showed reactive astroglial cells $\left(\mathrm{GFAP}^{+}\right.$cells) in the lesion site. These reactive astrocytes abundantly expressed proinflammatory markers such as Cox2 (Fig. 3B,C) and iNOS2 (Fig. 3C). In addition, Doxtreated AIC mice show detectable levels of the reactive microglia marker MHCII, as well as the presence of $\mathrm{MHCII}^{+}$and $\mathrm{CD} 11 \mathrm{~b}^{+}$ cells in the lesioned area (Fig. 3D). Notably, MHCII levels, as well as $\mathrm{MHCII}^{+}$and double-stained $\mathrm{MHCII}{ }^{+} / \mathrm{CD} 11 \mathrm{~b}^{+}$cells, but not $\mathrm{CD}_{11 \mathrm{~b}^{+}}$cells that remained elevated, were significantly attenuated in AIC mice expressing $\Delta \mathrm{CnA}$ before injury $(p<0.001$ vs + Dox AIC mice; $n=10$ ) (Fig. $3 D,-$ Dox) (supplemental Fig. $2 A$, available at www.jneurosci.org as supplemental material). At $5 \mathrm{~d}$ after lesion, the number of apoptotic neurons in the lesioned area was significantly decreased when $\Delta \mathrm{CnA}$ was expressed: 
A
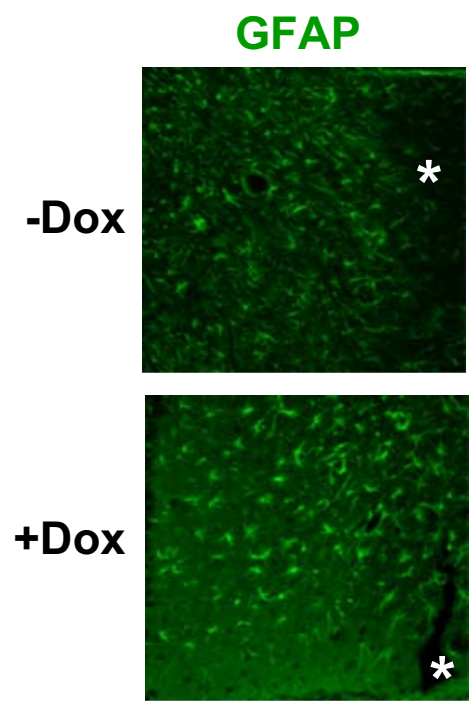

*
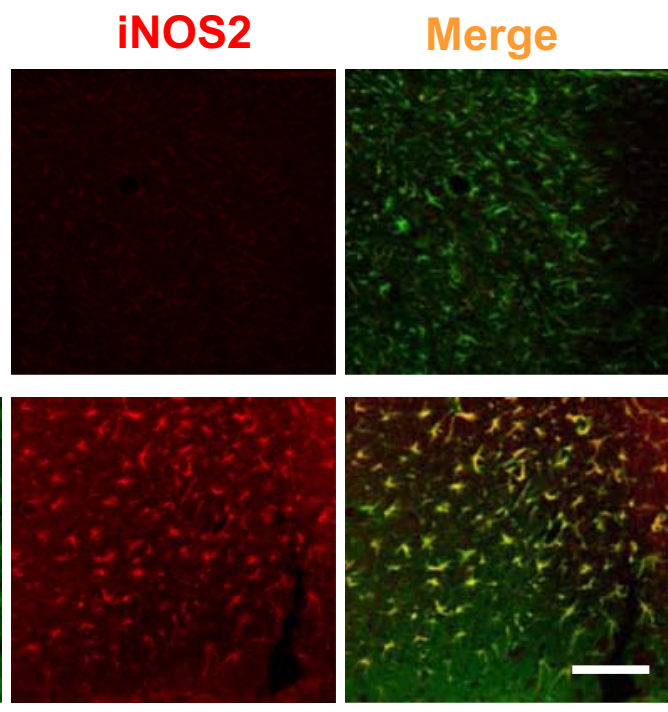

B
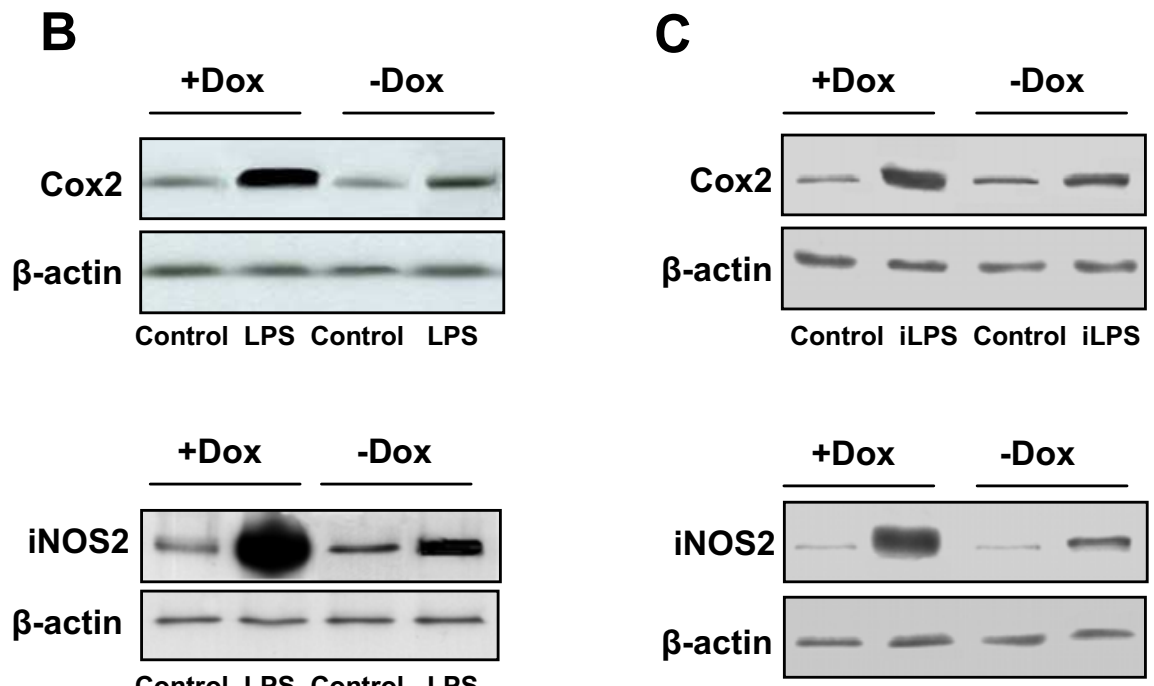

Control LPS Control LPS

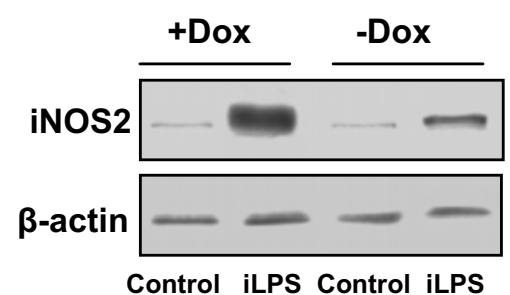

Figure 4. Astrocytic calcineurin protects against LPS-induced inflammatory damage. $\boldsymbol{A}$, Three days after intraparenchymael injection of $L P S$, reactive astrocytes (GFAP ${ }^{+}$) in AIC mice not treated with Dox show negligible iNOS2 immunoreactivity. Scale bar, $100 \mu \mathrm{m} . \boldsymbol{B}$, This was paralleled by a drastic reduction in levels of Cox2 and iNOS2 in the injected area ( $p<0.001$ vs AIC + Dox; $n=10)$. C, Levels of Cox2 and iNOS2 in cortical tissue of intraperitoneally LPS (iLPS)-injected AIC ( - Dox) mice were reduced compared with AIC mice receiving Dox in the drinking water ( $p<0.001$ vs AIC + Dox; $n=10$ at $3 \mathrm{~d}$ after injection). Representative blots are shown.

$20.6 \pm 1.0 \beta 3$-tubulin ${ }^{+}$-activated caspase $3^{+}$cells $/ \mathrm{mm}^{2}$ in - Dox vs $82.6 \pm 1.7$ cells $/ \mathrm{mm}^{2}$ in + Dox AIC mice $(p<0.001 ; n=10)$. Screening of proinflammatory protein levels with a protein array at day 5 after lesion (Raybiotech) (supplemental Results, available at www.jneurosci.org as supplemental material) confirmed a general downregulation by $\Delta \mathrm{CnA}$ expression (Table 1). Microglia reactivity and macrophage infiltration at the damaged site, determined by levels of MHCII and number of $\mathrm{MHCII}^{+}$and $\mathrm{CD}_{11 \mathrm{~b}^{+}}$cells, was also attenuated (Fig. 3D). Furthermore, as seen in vitro, the number and the intensity of staining of IGF-Iimmunoreactive astrocytes (IGF-I ${ }^{+} / \mathrm{GFAP}^{+}$cells) in the vicinity of the lesion was greater in AIC mice expressing $\triangle \mathrm{CnA}$ (from $83 \pm 16$ astrocytes $/ \mathrm{mm}^{2}$ in + Dox to $102 \pm 27$ astrocytes $/ \mathrm{mm}^{2}$ in - Dox; $p<0.05)$. Notably, when expression of $\Delta \mathrm{CnA}$ was al- lowed in astrocytes after brain trauma, once the inflammatory cascade is active, neuronal death was still markedly attenuated. Thus, in AIC mice in which Dox treatment was discontinued $2 \mathrm{~d}$ after injury ( \pm Dox mice) to allow $\Delta$ CnA expression (that starts within $24 \mathrm{~h}$ of Dox withdrawal), the number of apoptotic neurons $5 \mathrm{~d}$ after injury was markedly reduced, close to the reduction seen when $\Delta \mathrm{CnA}$ expression was allowed before injury (-Dox mice). There were $32 \pm 0.7$ apoptotic neurons $/ \mathrm{mm}^{2}$ in \pm Dox, $17 \pm 2.2$ neurons in - Dox, but $75 \pm 5.3$ neurons in + Dox AIC mice $(p<0.001$ vs \pm Dox and -Dox groups; $n=5$ per group). In \pm Dox AIC mice, microglia reactivity was also significantly reduced (Fig. 3D, bottom histograms) ( $p<0.001$ vs + Dox group).

Injection of bacterial endotoxins (LPS) into the brain is a well established model of inflammatory processes associated with human neurotropic infections (Fassbender et al., 2004; Felts et al., 2005). After brain LPS injection to AIC mice, pathological alterations peaked within $1-3 \mathrm{~d}$ and were resolved $10 \mathrm{~d}$ later. We examined the response to $\triangle \mathrm{CnA}$ expression $3 \mathrm{~d}$ after LPS challenge and found an attenuated inflammatory response when $\triangle \mathrm{CnA}$ was expressed. Significantly reduced neuronal death after LPS injection was seen in AIC mice without Dox before endotoxic challenge: $12.0 \pm 1.8 \beta 3$-tubulin ${ }^{+} /$activated caspase $3^{+}$cells $/ \mathrm{mm}^{2}$ in - Dox vs $63.3 \pm$ $1.0 \beta 3$-tubulin ${ }^{+} /$activated caspase $3^{+}$ cells $/ \mathrm{mm}^{2}$ in + Dox AIC mice $(p<0.001$; $n=10)$. As seen after brain trauma, markers of glial reactivity were also reduced in $\triangle \mathrm{CnA}$-expressing AIC mice. These included reduced activation of astrocytes $\left(\mathrm{GFAP}^{+} / \mathrm{iNOS}^{+}\right)$(Fig. $4 A$ ), decreased levels of proinflammatory mediators in the lesioned area $(p<0.001$ vs AIC + Dox; $n=10$ ) (Fig. $4 B$, Table 1) (supplemental Fig. $2 B$, available at www.jneurosci.org as supplemental material), and reduced microglia activation (data not shown). Furthermore, systemic administration of LPS to mimic central inflammatory responses to infection, a common complication in elderly demented patients (Perry et al., 2003), resulted also in an attenuated brain inflammatory response when AIC mice expressed $\triangle \mathrm{CnA}$. Five days after intraperitoneal injection of LPS, the levels of Cox 2 and iNOS2 in cortical tissue of AIC (-Dox) mice were reduced compared with AIC mice receiving Dox in the drinking water (Fig. 4C) (supplemental Fig. $2 C$, available at www.jneurosci.org as supplemental material $)(p<0.001$ vs AIC +Dox; $n=10$ ).

Calcineurin inhibits a canonical proinflammatory pathway in astrocytes

We then explored mechanisms underlying reduced inflammatory responses when calcineurin is activated in astrocytes before 
inflammatory challenge. In these studies, we used an in vitro approach to determine mechanisms involved at the molecular level. First, we validated the system by determining whether expression of $\Delta \mathrm{CnA}$ in cultured rat astrocytes also ameliorated the subsequent response to inflammatory challenge. Indeed, as seen in vivo in AIC mice, astrocytes expressing $\Delta \mathrm{CnA}$ before inflammatory challenge show reduced increases of the inflammatory effectors Cox2 and iNOS2 $16 \mathrm{~h}$ after LPS (Fig. $5 A$ ) or $3 \mathrm{~h}$ after TNF- $\alpha$ (data not shown). Other proinflammatory signals were also inhibited (Table 1). With this system, we next observed that, in $\Delta \mathrm{CnA}$-transfected astrocytes, but not in mock-transfected ones, LPS- or TNF- $\alpha$-induced activation of $\mathrm{NF} \kappa \mathrm{B}$ and NFAT was inhibited even below basal levels ( $p<0.001$ vs CMV astrocytes) (Fig. $5 B$ ). This inhibitory effect of $\Delta \mathrm{CnA}$ was not seen in neurons (supplemental Fig. $3 A$, available at www.jneurosci.org as supplemental material). Confirming these results in rat astrocytes, we found that $\mathrm{NF} \kappa \mathrm{B}$ and NFAT activity after inflammatory challenge was again inhibited below basal levels in astrocytes from AIC mice expressing $\Delta \mathrm{CnA}$, i.e., cultured in the absence of Dox (Fig. 5C).

Mechanisms whereby calcineurin may block the activation of NF $\kappa \mathrm{B}$ and NFAT by proinflammatory stimuli were then analyzed. As seen previously after IGF-Iinduced activation of calcineurin (Pons and Torres-Aleman, 2000), expression of $\Delta \mathrm{CnA}$ in astrocytes blocked phosphorylation of $\mathrm{I} \kappa \mathrm{B} \alpha$ by TNF- $\alpha$ (Fig. $5 D$ ). Furthermore, expression of $\triangle \mathrm{CnA}$ blocked the increase in the levels of $\mathrm{NF} \kappa \mathrm{Bp} 65$ and NFAT4 (an isoform found in astrocytes) (Jones et al., 2003) elicited by $16 \mathrm{~h}$ of exposure to LPS or $3 \mathrm{~h}$ after TNF- $\alpha$ (data not shown) in mock-transfected astrocytes (Fig. 5E) (supplemental Fig. 3B) $(p<$ 0.001 vs CMV-transfected astrocytes; $n=$ 6). We next explored mechanism whereby expression of $\Delta \mathrm{CnA}$ results in reduced levels of NF $\kappa$ Bp 65 and NFAT4 after inflammatory challenge. First, we inhibited the proteasome pathway with MG-132 and found that the effect of $\Delta \mathrm{CnA}$ was blocked (Fig. 5F) (supplemental Fig. 3C, available at www.jneurosci.org as supplemental material) ( $p<0.001$ vs $\Delta$ CnA astrocytes; $n=$ $6)$. This suggested that the proteasome pathway is involved in the reduction of the levels of NF $\kappa$ Bp 65 and NFAT4 produced by $\Delta \mathrm{CnA}$. In agreement with this possibility, we observed that $\Delta \mathrm{CnA}$-expressing astrocytes had increased NF $\kappa$ Bp 65 and NFAT4 complexed with ubiquitin, a protein tag to the proteasome pathway ( $p<0.001$ vs CMV-transfected astrocytes; $n=6$ ) (Fig. 5G) (supplemental Fig. 3D, available at www.
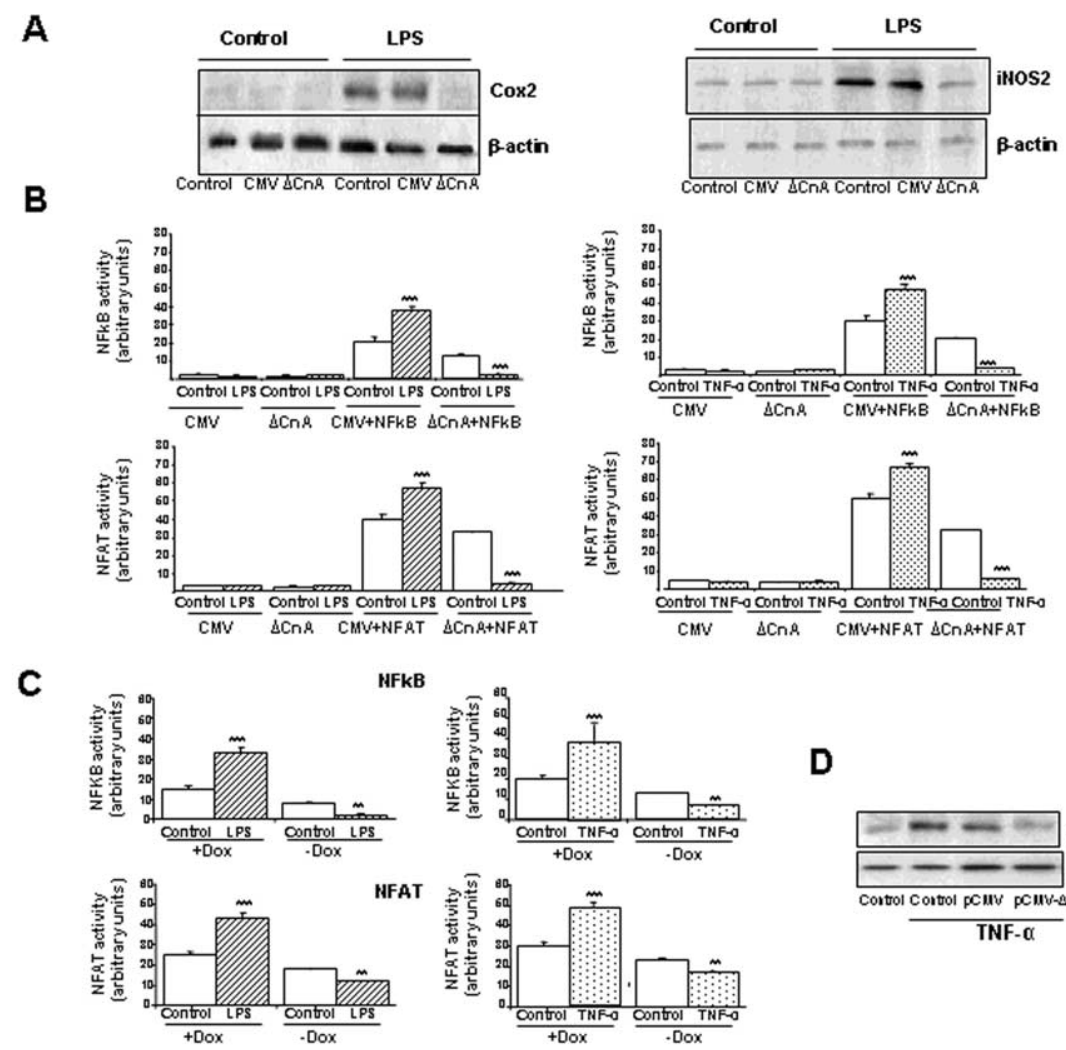

D
E

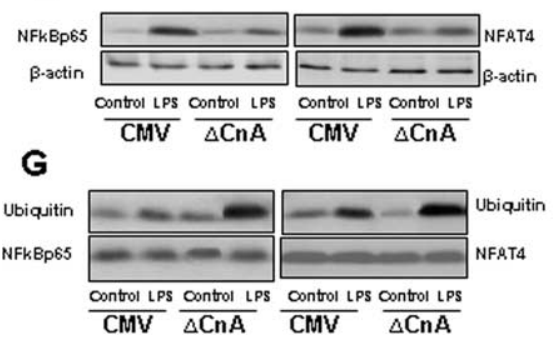

$\mathbf{F}$

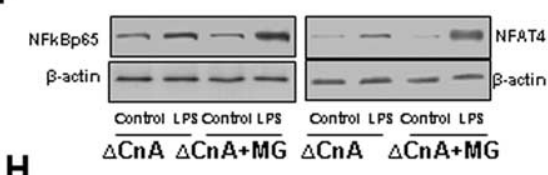

H

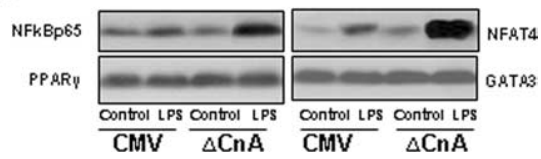

Figure 5. Calcineurin inhibits the NF $\kappa$ B/NFAT proinflammatory pathway in astrocytes. $A$, LPS failed to stimulate Cox2 and iNOS2 in astrocytes expressing $\Delta C \mathrm{CnA}$. A representative blot is shown $(n=6) . \boldsymbol{B}$, LPS- or TNF- $\alpha$-induced activation of NF $\kappa$ B and NFAT was abrogated in $\Delta$ CnA-transduced astrocytes. The activity of these transcription factors was inhibited after exposure to LPS/TNF- $\alpha$ in $\Delta$ CnA-expressing astrocytes. Note that expression of $\Delta C n A$ in unstimulated astrocytes reduced the activity of NF $\kappa B$ and NFAT. CMV, Astrocytes transfected with the empty vector; $\triangle C n A$, astrocytes transfected with constitutively active calcineurin; CMV +NF $\kappa B$ or NFAT, mock-transfected astrocytes expressing the gene-reporter system for either transcription factor; $\Delta \mathrm{CnA}+\mathrm{NF} \kappa \mathrm{B}$ or NFAT, $\Delta \mathrm{CnA}$-transfected astrocytes expressing the gene-reporter system. ${ }^{* *} p<0.001$ versus respective controls $(n=6)$. C, In astrocytes from AIC mice, activation of NF $\kappa$ B and NFAT after LPS (left) or TNF- $\alpha$ (right) was regulated by Dox, confirming that expression of $\Delta$ CnA suppresses it. ${ }^{* *} p<0.01$ and ${ }^{* * *} p<0.001$ versus respective controls. $\boldsymbol{D}$, Expression of $\Delta$ CnA blocks phosphorylation of $\mathrm{I}_{\kappa} B \alpha$ induced by TNF- $\alpha(20 \mathrm{ng} / \mathrm{ml}, 15 \mathrm{~min}$ of exposure, representative blot). Mock-transfected cultures received the pCMV vector. $\boldsymbol{E}$, Exposure of mock-transfected astrocytes to LPS elicited an increase in NF $\kappa$ Bp65 (left) and NFAT4 (right) protein levels $16 \mathrm{~h}$ later. This increase was significantly attenuated in astrocytes expressing $\Delta C$ CnA. $F$, The inhibitory effect of $\Delta$ CnA on NF $\kappa$ Bpp65/NFAT4 levels was blocked by the proteasome inhibitory drug MG-132 (MG). G, $\Delta$ CnA-expressing astrocytes have significantly increased NF $\kappa$ Bp65 and NFAT4 complexed with ubiquitin. Immunoprecipitation with anti-NF $\kappa$ Bp65 or anti-NFAT4 followed by blotting with anti-ubiquitin (top blots). $\boldsymbol{H}$, Astrocytes transduced with $\Delta$ CnA had increased levels of $\mathrm{NF} \kappa \mathrm{Bp} 65 / \mathrm{PPAR} \gamma$ and NFAT/GATA3 compared with control astrocytes. Immunoprecipitation with anti-PPAR $\gamma$ and anti-GATA3 followed by blotting with either anti-NF $\kappa$ Bp65 or anti-NFAT4 (top blots). The opposite immunoprecipitations gave similar results (data not shown). In all cases, levels of $\beta$-actin or the respective immunoprecipitated protein were determined for protein load (bottom blots). Representative blots (of 6 for each experiment) are shown. All changes were significantly different (see Results).

jneurosci.org as supplemental material). At the same time, the inhibitory action of $\Delta \mathrm{CnA}$ was accompanied by increased association of NFAT4 with GATA3 (an isoform of brain tissue) (Pandolfi et al., 1995) and of NF $\kappa$ Bp65 with PPAR $\gamma$ (Fig. 5H) 
A
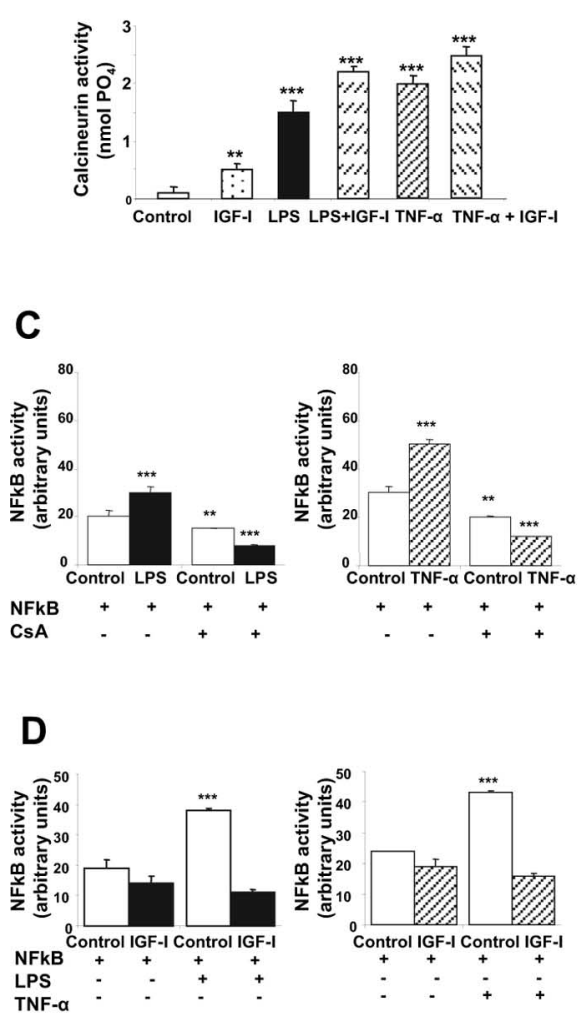

E

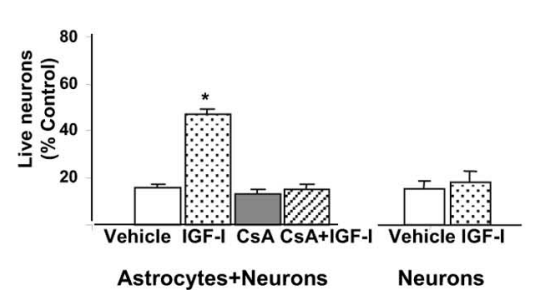

B

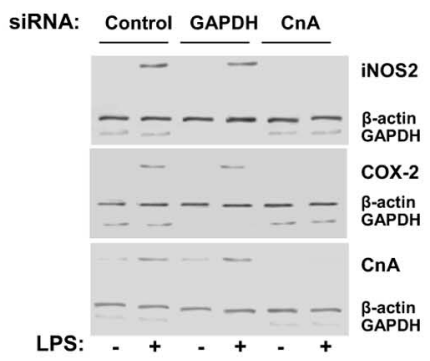

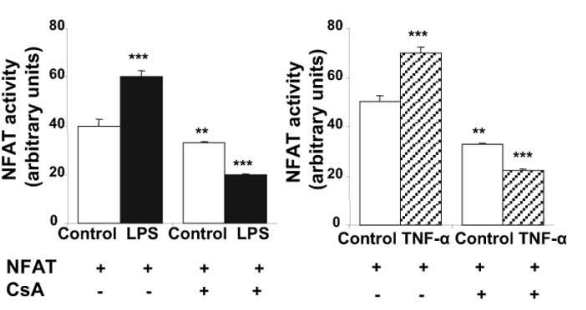

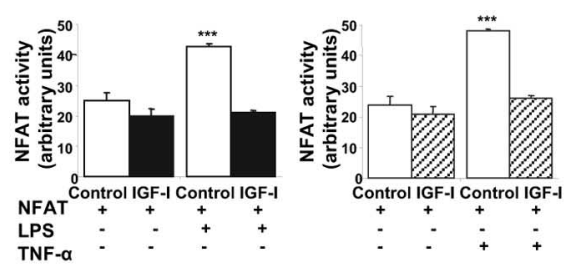

$\mathbf{F}$

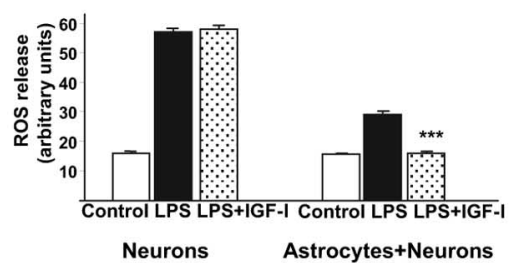

Figure 6. Astrocytic calcineurin participates in proinflammatory and in anti-inflammatory signaling. $\boldsymbol{A}$, In wt astrocytes, calcineurin activity was incremented by proinflammatory stimuli such as LPS/TNF- $\alpha$ and by neuroprotective signals such as IGF-I. ${ }^{* *} p<0.01$ and ${ }^{* * *} p<0.001$ versus control. $\boldsymbol{B}$, In astrocytes transfected with a calcineurin-specific siRNA (CnA3), the production of inflammatory mediators such as iNOS2 or Cox2 in response to inflammatory challenge (LPS) was abrogated in parallel to inhibited calcineurin levels. Controls, Astrocytes transfected with non-sense siRNA; GAPDH, astrocytes transfected with GAPDH siRNA. Note that, although levels of this protein are depleted, the response to LPS/TNF- $\alpha$ remains intact. Representative blots are shown $(n=6)$. Other $C n A$ siRNAs tested gave similar results (data not shown). C, LPS/TNF- $\alpha$-stimulated activity of NF $\kappa$ B and NFAT was abrogated in the presence of CSA. NF $\kappa B$ and NFAT denote transfection of astrocytes with NF $\kappa B$ and NFAT reporters, respectively. ${ }^{* *} p<0.01$ and ${ }^{* * *} p<0.001$ versus untreated controls (shown in left white bars). $\boldsymbol{D}$, IGF-I inhibited the increase induced by LPS or TNF- $\alpha$ in the activity of NF $\kappa$ B and NFAT. ${ }^{* * *} p<0.001$ versus unstimulated controls $(n=6) .{ }^{* * *} p<0.001$ versus unstimulated controls. $\boldsymbol{E}$, IGF-I protected neurons against TNF- $\alpha$-induced death only in the presence of astrocytes. This effect was blocked by CSA. Neurons were exposed to TNF- $\alpha$ overnight. $\boldsymbol{F}$, Production of ROS after LPS challenge was blocked by IGF-I only in the presence of astrocytes. ${ }^{*} p<0.05$ and ${ }^{* * *} p<0.001$ versus respective controls $(n=6)$.

(supplemental Fig. 3E, available at www.jneurosci.org as supplemental material) $(p<0.001$ vs CMV astrocytes; $n=6)$. These two transcription factors modulate the activity of $\mathrm{NF} \kappa \mathrm{B}$ and NFAT, respectively (Avni et al., 2002; Bailey and Ghosh, 2005). Similar changes were seen in astrocytes obtained from AIC mice and cultured in the presence or absence of Dox (data not shown).

\section{Astrocyte calcineurin is recruited by both proinflammatory and anti-inflammatory signals}

Although our results indicated that active calcineurin counteracts a subsequent inflammatory stimulus, this phosphatase is known to participate in inflammatory responses (Staruch et al., 1998). Indeed, as seen in other cell types, in quiescent wt astrocytes, calcineurin is activated by LPS and TNF- $\alpha$ (Fig. 6A). Moreover, when endogenous calcineurin is depleted in wt astrocytes by transfection of an siRNA against calcineurin, the production of inflammatory mediators such as iNOS2 or Cox2 in response to LPS is abrogated (Fig. 6B). LPS and TNF- $\alpha$ also stimulated the activity of the proinflammatory transcription factors $\mathrm{NF} \kappa \mathrm{B}$ and NFAT and in a calcineurindependent manner, as evidenced by its blockade with CsA (Fig. 6C).

However, an anti-inflammatory factor such as IGF-I, which inhibits TNF- $\alpha$ stimulated NF $\kappa$ B in astrocytes (Pons and Torres-Aleman, 2000), also enhanced calcineurin activity in wt astrocytes (Fig. 6A). At the same time, IGF-I was able to inhibit the increase in NF $\kappa \mathrm{B}$ and NFAT activity after inflammatory stimuli (Fig. 6D). That activation of calcineurin in astrocytes is essential for the neuroprotective actions of IGF-I against inflammation was evidenced by the fact that IGF-I rescued neurons of TNF- $\alpha$-induced death (Fig. $6 E$ ) or block neuronal production of ROS after TNF- $\alpha$ (Fig. 6F) only when astrocytes were present. A similar protective effect of IGF-I was seen after LPS (data not shown). Furthermore, calcineurin was essential for IGF-I neuroprotection because cyclosporine A inhibited its actions (Fig. 6E). Because IGF-I is found in reactive astrocytes in brain lesions in which inflammation occurs (supplemental Fig. 3F, available at www.jneurosci.org as supplemental material), it may activate astrocyte calcineurin during brain injury.

\section{Discussion}

Glial cells are a rich source of trophic signals for neurons, and its potential use as therapeutic targets in neurodegenerative diseases has already been outlined (Ranaivo et al., 2006). However, a better knowledge of the pathways involved in glia-to-neuron protective communication is needed before we can properly address this goal. Our present observations indicate that the phosphatase calcineurin in astrocytes modulates neuronal damage associated with inflammation, a common condition in the neurodegenerative process (Minghetti, 2005). Intriguingly, in brain diseases in which inflammation is involved such as Alzheimer's disease (Mrak and Griffin, 2005) or amyotrophic lateral sclerosis (Weydt et al., 2002), calcineurin activity has been reported to be abnormally low (Ladner et al., 1996; Ferri et al., 2001), whereas in situ generation of constitutively active calcineurin is neuroprotective in brain ischemia (Shioda et al., 2006).

Although the role of calcineurin in inflammation has long 
been known and its inhibitors are used in the clinic (Shanley, 2002), its concomitant involvement in anti-inflammatory signaling was not documented (Fig. 7). This ambivalent mechanism of astrocyte calcineurin, reminiscent of that reported for CD14 in immune cell responses (Devitt et al., 1998), may help explain the long-held notion that an interplay between proinflammatory and anti-inflammatory signals dictates the outcome of the inflammatory process and, ultimately, its associated neuronal loss (Wyss-Coray and Mucke, 2002). Indeed, signals that activate calcineurin, including neuroprotective factors such as IGF-I, together with cytokines such as TNF- $\alpha$ are present in inflammatory lesions in a distinct time-dependent manner. Whereas TNF- $\alpha$ increases within hours after damage (Yin et al., 2003), IGF-I levels peak after days (Beilharz et al., 1998). This pattern of activation would theoretically allow a calcineurindependent termination of the acute inflammatory phase and resultant generation of ROS by an anti-inflammatory action of IGF-I and other protective signals. Other signals are clearly involved because inhibition of the action of calcineurin-induced IGF-I is not sufficient to abrogate the neuroprotective actions of calcineurin-expressing astrocytes. Among these, SOD appears as a likely candidate because its production was enhanced, and this can be related to ROS inhibition and neuroprotection because SOD is a potent free radicals inhibitor and neuroprotective signal.

In initial stages of the inflammatory process, calcineurin will be activated by TNF- $\alpha$ (and other mediators of inflammation). Once IGF-I (and other neuroprotective mediators such as SOD) produced mostly by activated microglia (and other local, i.e., astrocytes, and peripheral sources) start to accumulate at the lesion site, calcineurin will be further stimulated (Fig. 6A). This IGF-I activation of already active calcineurin would result in cancellation of the inflammatory cascade and potentiation of the neuroprotective pathway (Fig. 7). The fact that transgenic expression of constitutively active calcineurin both in vivo and in vitro results also in neuroprotection suggests that a certain level of calcineurin activity (compare the levels of active calcineurin in Figs. $1 A$ and $6 A$ ) is required for neuroprotection. Although the initial calcineurin-dependent release of proinflammatory mediators after insult by astrocytes is considered to be beneficial, its subsequent harnessing by calcineurindependent anti-inflammatory pathways may be critical to avoid maladaptive responses.

Previous reports indicated both beneficial and detrimental actions of calcineurin in the response of astrocytes to insults, including inflammation (Pons and Torres-Aleman, 2000; Norris et al., 2005). Gene expression analysis of mixed astrocyte-neuronal cultures transduced with $\Delta \mathrm{CnA}$ showed a reactive/inflammatory astrocyte phenotype. However, expression of $\Delta \mathrm{CnA}$ in

\section{Initiation}

nflammatory challenge

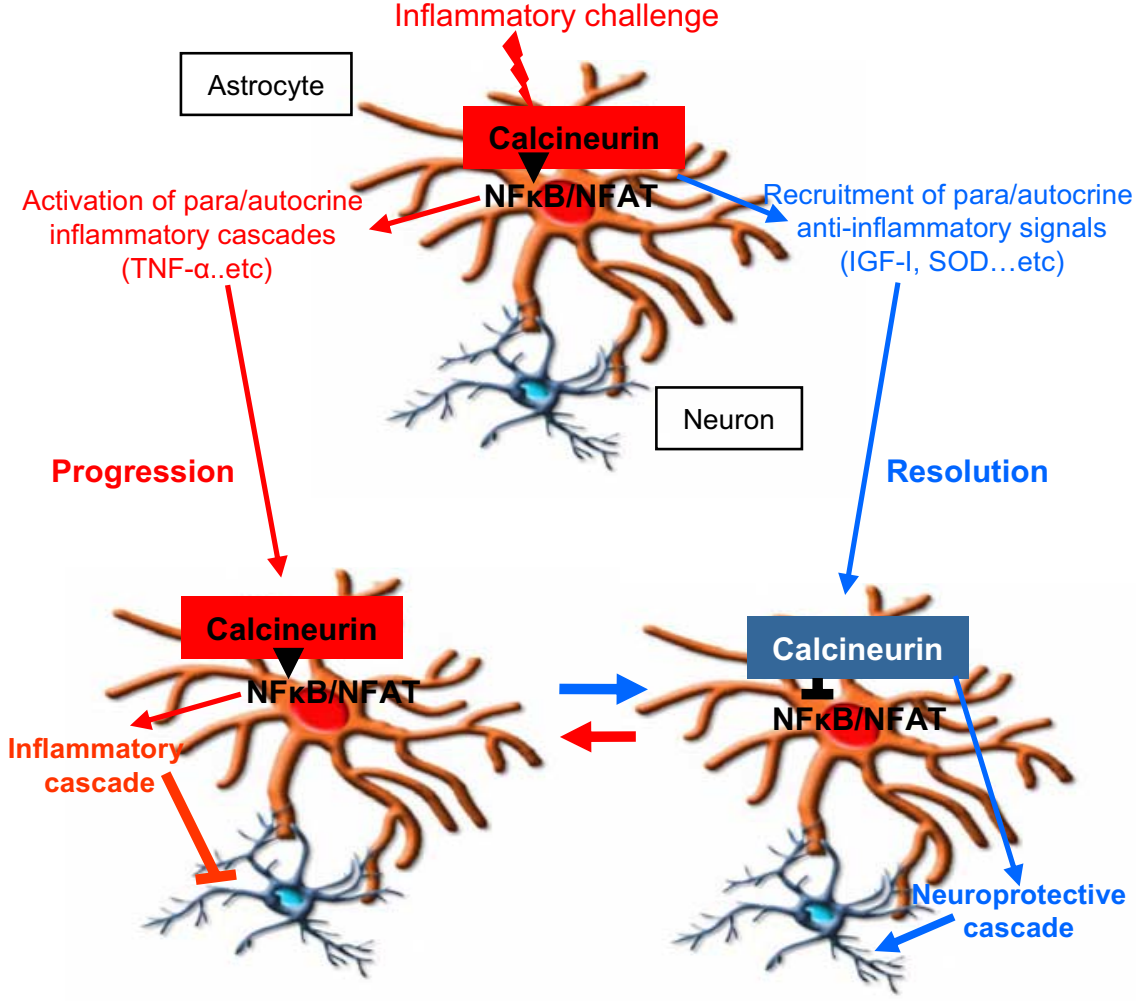

Figure 7. Stages of the neuroinflammatory process in which astrocyte calcineurin may participate. Initiation, Inflammatory in peurin is recruited toward either inflammation or neuroprotection, which involve differential interactions with transcription factors such as PPAR $\gamma$ and GATA3 or proteasome degradation and which depend on the upstream signal stimulating calcineurin, warrant additional analysis.

mixed cultures also induced neuroprotective signals, and neuronal proapoptotic genes were downregulated (Norris et al., 2005) Collectively, it seems that activation of calcineurin in resting astrocytes leads to an inflammatory phenotype, whereas its activation in stimulated astrocytes (after stimuli such as inflammatory mediators) results in abrogation of inflammatory cascades. This cell-context-dependent pattern of calcineurin signaling, a common feature of intracellular signaling pathways (Natarajan et al., 2006), may help understand the apparently contradictory observation that calcineurin can regulate NFAT/NF $\kappa$ B activity in opposite ways (Rao et al., 1997; Biswas et al., 2003; Kim et al., 2004; Martinez-Martinez et al., 2006). Calcineurin is also known to play opposing roles in apoptotic responses, depending on cell context and type of stimulus. Indeed, modulation of calcineurin in astrocytes affects their response to apoptotic stimuli (Kaminska et al., 2004), which could theoretically explain its neuroprotective effect because astrocytes are essential protective partners for neurons (Takuma et al., 2004). However, the fact that the proinflammatory stimuli used in the present study did not produce astrocyte death makes it unlikely that astrocyte calcineurin protected neurons by modulating astrocyte apoptosis. 
Although the routes involved in stimulation of NFAT and $\mathrm{NF} \kappa \mathrm{B}$ are well described, the pathways involved in their inactivation are less known. The present results suggest that calcineurin inhibits NF $\kappa$ B by dephosphorylation of nuclear factor $\kappa \mathrm{B}$ inhibitor $(\mathrm{I} \kappa \mathrm{B})$ and interactions with PPAR $\gamma$, and NFAT through interactions with GATA. Both transcription factors have been shown previously to interact with NFAT and NF $\kappa$ B, respectively (Chinetti et al., 1998; Wada et al., 2002). Intriguingly, recent evidence indicates that not only calcineurin (see above) but also PPAR $\gamma$ activity is diminished in Alzheimer's disease patients (Sastre et al., 2006). Calcineurin may also direct NFAT and NF $\kappa$ B to the proteasome pathway because lower levels of both transcription factors together with increased association to ubiquitin was observed in the presence of $\Delta \mathrm{CnA}$. Our results also confirm the stimulatory role of calcineurin on neuroprotective signals such as SOD and IGF-I (McCall et al., 2003; Norris et al., 2005), providing a conceptual framework to understand the contribution of astroglia to neuronal death in diseases such as amyotrophic lateral sclerosis (Clement et al., 2003), in which these neuroprotective signals are involved (Wilczak et al., 2003; Turner et al., 2005).

Altogether, it seems that calcineurin plays a dual role in activating proinflammatory cascades in response to proinflammatory signals such as TNF- $\alpha$ or canceling them and at the same time promoting neuroprotection in response to trophic signals such as IGF-I. At any rate, the pathways underlying this dual action of calcineurin, which probably involves interactions with scaffolding and modulatory proteins (Klauck et al., 1996; Fuentes et al., 2000; Abbasi et al., 2006), and release of various neuromodulators warrant additional studies.

In summary, astrocyte calcineurin may constitute a key element in the process whereby an acute inflammatory response progresses toward a neuroprotective effect and is resolved or develops into a sustained insult.

\section{References}

Abbasi S, Lee JD, Su B, Chen X, Alcon JL, Yang J, Kellems RE, Xia Y (2006) Protein kinase-mediated regulation of calcineurin through the phosphorylation of modulatory calcineurin-interacting protein 1. J Biol Chem 281:7717-7726.

Alfieri C, Evans-Anderson H, Yutzey K (2007) Developmental regulation of the mouse IGF-I exon 1 promoter region by calcineurin activation of NFAT in skeletal muscle. Am J Physiol Cell Physiol 292:C1887-C1894.

Avni O, Lee D, Macian F, Szabo SJ, Glimcher LH, Rao A (2002) T(H) cell differentiation is accompanied by dynamic changes in histone acetylation of cytokine genes. Nat Immunol 3:643-651.

Bailey ST, Ghosh S (2005) "PPAR"ting ways with inflammation. Nat Immunol 6:966-967.

Beilharz EJ, Russo VC, Butler G, Baker NL, Connor B, Sirimanne ES, Dragunow M, Werther GA, Gluckman PD, Williams CE, Scheepens A (1998) Co-ordinated and cellular specific induction of the components of the IGF/IGFBP axis in the rat brain following hypoxic-ischemic injury. Brain Res Mol Brain Res 59:119-134.

Biswas G, Anandatheerthavarada HK, Zaidi M, Avadhani NG (2003) Mitochondria to nucleus stress signaling: a distinctive mechanism of NFkap$\mathrm{paB} /$ Rel activation through calcineurin-mediated inactivation of IkappaBbeta. J Cell Biol 161:507-519.

Brera B, Serrano A, de Ceballos ML (2000) beta-Amyloid peptides are cytotoxic to astrocytes in culture: a role for oxidative stress. Neurobiol Dis 7:395-405.

Chinetti G, Griglio S, Antonucci M, Torra IP, Delerive P, Majd Z, Fruchart JC, Chapman J, Najib J, Staels B (1998) Activation of proliferator-activated receptors alpha and gamma induces apoptosis of human monocytederived macrophages. J Biol Chem 273:25573-25580.

Clement AM, Nguyen MD, Roberts EA, Garcia ML, Boillee S, Rule M, McMahon AP, Doucette W, Siwek D, Ferrante RJ, Brown Jr RH, Julien JP, Goldstein LS, Cleveland DW (2003) Wild-type nonneuronal cells ex- tend survival of SOD1 mutant motor neurons in ALS mice. Science 302:113-117.

Devitt A, Moffatt OD, Raykundalia C, Capra JD, Simmons DL, Gregory CD (1998) Human CD14 mediates recognition and phagocytosis of apoptotic cells. Nature 392:505-509.

Fassbender K, Walter S, Kühl S, Landmann R, Ishii K, Bertsch T, Stalder AK, Muehlhauser F, Liu Y, Ulmer AJ, Rivest S, Lentschat A, Gulbins E, Jucker M, Staufenbiel M, Brechtel K, Walter J, Multhaup G, Penke B, Adachi Y, Hartmann T, Beyreuther K (2004) The LPS receptor (CD14) links innate immunity with Alzheimer's disease. FASEB J 18:203-205.

Felts PA, Woolston AM, Fernando HB, Asquith S, Gregson NA, Mizzi OJ, Smith KJ (2005) Inflammation and primary demyelination induced by the intraspinal injection of lipopolysaccharide. Brain 128:1649-1666.

Fernandez AM, Garcia-Estrada J, Garcia-Segura LM, Torres-Aleman I (1997) Insulin-like growth factor I modulates c-Fos induction and astrocytosis in response to neurotoxic insult. Neuroscience 76:117-122.

Fernandez AM, de la Vega AG, Torres-Aleman I (1998) Insulin-like growth factor I restores motor coordination in a rat model of cerebellar ataxia. Proc Natl Acad Sci USA 95:1253-1258.

Fernandez AM, Kim JK, Yakar S, Dupont J, Hernandez-Sanchez C, Castle AL, Filmore J, Shulman GI, Le Roith D (2001) Functional inactivation of the IGF-I and insulin receptors in skeletal muscle causes type 2 diabetes. Genes Dev 15:1926-1934.

Ferri A, Gabbianelli R, Casciati A, Celsi F, Rotilio G, Carri MT (2001) Oxidative inactivation of calcineurin by $\mathrm{Cu}, \mathrm{Zn}$ superoxide dismutase G93A, a mutant typical of familial amyotrophic lateral sclerosis. J Neurochem 79:531-538.

Fuentes JJ, Genesca L, Kingsbury TJ, Cunningham KW, Perez-Riba M, Estivill $\mathrm{X}$, de la LS (2000) DSCR1, overexpressed in Down syndrome, is an inhibitor of calcineurin- mediated signaling pathways. Hum Mol Genet 9:1681-1690.

Ginis I, Jaiswal R, Klimanis D, Liu J, Greenspon J, Hallenbeck JM (2002) TNF-alpha-induced tolerance to ischemic injury involves differential control of NF-kappaB transactivation: the role of NF-kappaB association with p300 adaptor. J Cereb Blood Flow Metab 22:142-152.

Jones EA, Sun D, Kobierski L, Symes AJ (2003) NFAT4 is expressed in primary astrocytes and activated by glutamate. J Neurosci Res 72:191-197.

Kaminska B, Gaweda-Walerych K, Zawadzka M (2004) Molecular mechanisms of neuroprotective action of immunosuppressants: facts and hypotheses. J Cell Mol Med 8:45-58.

Kim S, Domon-Dell C, Kang J, Chung DH, Freund JN, Evers BM (2004) Down-regulation of the tumor suppressor PTEN by the tumor necrosis factor-alpha/nuclear factor-kappaB (NF-kappaB)-inducing kinase/NFkappaB pathway is linked to a default IkappaB-alpha autoregulatory loop. J Biol Chem 279:4285-4291.

Klauck TM, Faux MC, Labudda K, Langeberg LK, Jaken S, Scott JD (1996) Coordination of three signaling enzymes by AKAP79, a mammalian scaffold protein. Science 271:1589-1592.

Ladner CJ, Czech J, Maurice J, Lorens SA, Lee JM (1996) Reduction of calcineurin enzymatic activity in Alzheimer's disease: correlation with neuropathologic changes. J Neuropathol Exp Neurol 55:924-931.

Lopez-Lopez C, LeRoith D, Torres-Aleman I (2004) Insulin-like growth factor I is required for vessel remodeling in the adult brain. Proc Natl Acad Sci USA 101:9833-9838.

Martinez-Martinez S, Rodriguez A, Lopez-Maderuelo MD, Ortega-Perez I, Vazquez J, Redondo JM (2006) Blockade of NFAT activation by the second calcineurin binding site. J Biol Chem 281:6227-6235.

McCall GE, Allen DL, Haddad F, Baldwin KM (2003) Transcriptional regulation of IGF-I expression in skeletal muscle. Am J Physiol Cell Physiol 285:C831-C839.

Minghetti L (2005) Role of inflammation in neurodegenerative diseases. Curr Opin Neurol 18:315-321.

Mrak RE, Griffin WS (2005) Glia and their cytokines in progression of neurodegeneration. Neurobiol Aging 26:349-354.

Natarajan M, Lin KM, Hsueh RC, Sternweis PC, Ranganathan R (2006) A global analysis of cross-talk in a mammalian cellular signalling network. Nat Cell Biol 8:571-580.

Norris CM, Kadish I, Blalock EM, Chen KC, Thibault V, Porter NM, Landfield PW, Kraner SD (2005) Calcineurin triggers reactive/inflammatory processes in astrocytes and is upregulated in aging and Alzheimer's models. J Neurosci 25:4649-4658.

O'Keefe SJ, Tamura J, Kincaid RL, Tocci MJ, O'Neill EA (1992) FK-506- and 
CsA-sensitive activation of the interleukin-2 promoter by calcineurin. Nature 357:692-694.

Pandolfi PP, Roth ME, Karis A, Leonard MW, Dzierzak E, Grosveld FG, Engel JD, Lindenbaum MH (1995) Targeted disruption of the GATA3 gene causes severe abnormalities in the nervous system and in fetal liver haematopoiesis. Nat Genet 11:40-44.

Perry VH, Newman TA, Cunningham C (2003) The impact of systemic infection on the progression of neurodegenerative disease. Nat Rev Neurosci 4:103-112.

Pons S, Torres-Aleman I (2000) Insulin-like growth factor-I stimulates dephosphorylation of ikappa B through the serine phosphatase calcineurin (protein phosphatase 2B). J Biol Chem 275:38620-38625.

Ranaivo HR, Craft JM, Hu W, Guo L, Wing LK, Van Eldik LJ, Watterson DM (2006) Glia as a therapeutic target: selective suppression of human amyloid- $\beta$-induced upregulation of brain proinflammatory cytokine production attenuates neurodegeneration. J Neurosci 26:662-670.

Rao A, Luo C, Hogan PG (1997) Transcription factors of the NFAT family: regulation and function. Annu Rev Immunol 15:707-747.

Ridet JL, Malhotra SK, Privat A, Gage FH (1997) Reactive astrocytes: cellular and molecular cues to biological function. Trends Neurosci 20:570-577.

Sastre M, Dewachter I, Rossner S, Bogdanovic N, Rosen E, Borghgraef P, Evert BO, Dumitrescu-Ozimek L, Thal DR, Landreth G, Walter J, Klockgether T, van LF, Heneka MT (2006) Nonsteroidal anti-inflammatory drugs repress beta-secretase gene promoter activity by the activation of PPARgamma. Proc Natl Acad Sci USA 103:443-448.

Seifert G, Schilling K, Steinhauser C (2006) Astrocyte dysfunction in neurological disorders: a molecular perspective. Nat Rev Neurosci 7:194-206.

Shanley TP (2002) Phosphatases: counterregulatory role in inflammatory cell signaling. Crit Care Med 30:S80-S88.

Shioda N, Moriguchi S, Shirasaki Y, Fukunaga K (2006) Generation of constitutively active calcineurin by calpain contributes to delayed neuronal death following mouse brain ischemia. J Neurochem 98:310-320.

Staruch MJ, Camacho R, Dumont FJ (1998) Distinctive calcineurin- dependent (FK506-sensitive) mechanisms regulate the production of the CC chemokines macrophage inflammatory protein (MIP)-1alpha, MIP1beta, and RANTES vs IL- 2 and TNF-alpha by activated human T cells. Cell Immunol 190:121-131.

Streit WJ, Walter SA, Pennell NA (1999) Reactive microgliosis. Prog Neurobiol 57:563-581.

Takuma K, Baba A, Matsuda T (2004) Astrocyte apoptosis: implications for neuroprotection. Prog Neurobiol 72:111-127.

Tokheim AM, Martin BL (2006) Association of calcineurin with mitochondrial proteins. Proteins 64:28-33.

Torres-Aleman I (2000) Serum growth factors and neuroprotective surveillance. Mol Neurobiol 21:153-160.

Trejo JL, Carro E, Torres-Aleman I (2001) Circulating insulin-like growth factor I mediates exercise-induced increases in the number of new neurons in the adult hippocampus. J Neurosci 21:1628-1634.

Turner BJ, Atkin JD, Farg MA, Zang DW, Rembach A, Lopes EC, Patch JD, Hill AF, Cheema SS (2005) Impaired extracellular secretion of mutant superoxide dismutase 1 associates with neurotoxicity in familial amyotrophic lateral sclerosis. J Neurosci 25:108-117.

Wada H, Hasegawa K, Morimoto T, Kakita T, Yanazume T, Abe M, Sasayama S (2002) Calcineurin-GATA-6 pathway is involved in smooth musclespecific transcription. J Cell Biol 156:983-991.

Weydt P, Weiss MD, Moller T, Carter GT (2002) Neuro-inflammation as a therapeutic target in amyotrophic lateral sclerosis. Curr Opin Investig Drugs 3:1720-1724.

Wilczak N, de Vos RA, De Keyser J (2003) Free insulin-like growth factor (IGF)-I and IGF binding proteins 2, 5, and 6 in spinal motor neurons in amyotrophic lateral sclerosis. Lancet 361:1007-1011.

Wyss-Coray T, Mucke L (2002) Inflammation in neurodegenerative disease: a double-edged sword. Neuron 35:419-432.

Yin L, Ohtaki H, Nakamachi T, Dohi K, Iwai Y, Funahashi H, Makino R, Shioda S (2003) Expression of tumor necrosis factor alpha (TNFalpha) following transient cerebral ischemia. Acta Neurochir Suppl 86:93-96. 